\title{
Interplay between Notch1 and Notch3 promotes EMT and tumor initiation in squamous cell carcinoma
}

\author{
Mitsuteru Natsuizaka1,2,3,4, Kelly A. Whelan²,2, Shingo Kagawa1,2,3,5, Koji Tanaka1,2,3,6, Veronique Giroux ${ }^{1,2,3}$, \\ Prasanna M. Chandramouleeswaran 1,2,3, Apple Long1,2,3, Varun Sahu2,3,7, Douglas S. Darling ${ }^{8}$, Jianwen Que ${ }^{9}$, \\ Yizeng Yang1,2,3, Jonathan P. Katz1,2,3, E. Paul Wileyto2,3,10, Devraj Basu'2,3, Yoshiaki Kita11, Shoji Natsugoe ${ }^{11}$, \\ Seiji Naganuma ${ }^{12}$, Andres J. Klein-Szanto ${ }^{13}$, J. Alan Diehl14, Adam J. Bass ${ }^{15}$, Kwok-Kin Wong ${ }^{15,16}$, Anil K. Rustgi ${ }^{3}$ \\ $\&$ Hiroshi Nakagawa ${ }^{3}$
}

Notch1 transactivates Notch3 to drive terminal differentiation in stratified squamous epithelia. Notch1 and other Notch receptor paralogs cooperate to act as a tumor suppressor in squamous cell carcinomas (SCCs). However, Notch1 can be stochastically activated to promote carcinogenesis in murine models of SCC. Activated form of Notch1 promotes xenograft tumor growth when expressed ectopically. Here, we demonstrate that Notch1 activation and epithelial-mesenchymal transition (EMT) are coupled to promote SCC tumor initiation in concert with transforming growth factor (TGF)- $\beta$ present in the tumor microenvironment. We find that TGF $\beta$ activates the transcription factor ZEB1 to repress Notch3, thereby limiting terminal differentiation. Concurrently, TGF $\beta$ drives Notch1-mediated EMT to generate tumor initiating cells characterized by high CD44 expression. Moreover, Notch1 is activated in a small subset of SCC cells at the invasive tumor front and predicts for poor prognosis of esophageal SCC, shedding light upon the tumor promoting oncogenic aspect of Notch1 in SCC.

\footnotetext{
${ }^{1}$ Gastroenterology Division, Department of Medicine, University of Pennsylvania, Philadelphia, PA 19104, USA. ${ }^{2}$ Abramson Cancer Center, Philadelphia, PA 19104, USA. ${ }^{3}$ University of Pennsylvania Perelman School of Medicine, Philadelphia, PA 19104, USA. ${ }^{4}$ Department of Gastroenterology and Hepatology, Hokkaido University Graduate School of Medicine, Sapporo, Hokkaido 060-8638, Japan. ${ }^{5}$ Department of General Surgery, Chiba University Graduate School of Medicine, Chiba, Chiba 260-0856, Japan. ${ }^{6}$ Department of Surgery, Gastroenterological Surgery, Osaka University Graduate School of Medicine, Suita, Osaka 565-0871, Japan. ${ }^{7}$ Department of Otorhinolaryngology, University of Pennsylvania, Philadelphia, PA 19104, USA. ${ }^{8}$ Department of Oral Immunology and Infectious Diseases, and Center for Genetics and Molecular Medicine, University of Louisville, Louisville, KY 40202, USA. ${ }^{9}$ Department of Medicine, Division of Digestive and Liver Diseases, Columbia University, New York, NY 10032, USA. ${ }^{10}$ Department of Biostatistics and Epidemiology, University of Pennsylvania, Philadelphia, PA 19104, USA. ${ }^{11}$ Department of Digestive Surgery, Breast and Thyroid Surgery, Kagoshima University Graduate School of Medical and Dental Sciences, Kagoshima 890-8520, Japan. ${ }^{12}$ Department of Pathology, Kochi Medical School, Nankoku-shi, Kochi 783-8505, Japan.

${ }^{13}$ Histopathology Facility and Cancer Biology Program, Fox Chase Cancer Center, Philadelphia, PA 19111, USA. ${ }^{14}$ Department of Biochemistry and Molecular Biology, Hollings Cancer Center, Medical University of South Carolina, Charleston, SC 29425, USA. ${ }^{15}$ Dana-Farber Cancer Institute, Department of Medicine, Harvard Medical School, Boston, MA 02215, USA. ${ }^{16}$ Division of Hematology and Medical Oncology, New York University, New York, NY 10016, USA. Mitsuteru Natsuizaka and Kelly A. Whelan contributed equally to this work. Correspondence and requests for materials should be addressed to K.-K.W. (email: kwok-kin.wong@nyumc.org) or to H.N. (email: nakagawh@mail.med.upenn.edu)
} 
$\mathrm{N}$ otch signaling regulates cell fate in a context-dependent manner ${ }^{1}$. The ligand-activated intracellular domain of Notch (ICN) forms a transcriptional activation complex with the transcription factor CSL and the co-activator MAML. Notch1 drives terminal differentiation in stratified squamous epithelia $^{2,3}$ in concert with other Notch receptor paralogs $s^{4,5}$. Histopathology of squamous cell carcinomas (SCCs) features squamous-cell differentiation, a process normally regulated via direct transcriptional activation of Notch 3 by ICN1, the activated form of Notch1, in esophageal epithelia ${ }^{4}$. Loss-of-function Notch1 mutations are found in SCCs ${ }^{6,7}$, suggesting a tumor suppressor role for Notch $1^{5,8}, 9$. However, Notch1 can be stochastically activated or inactivated, with either scenario resulting in promotion of carcinogenesis in murine models of $\mathrm{SCC}^{10}$. Many human SCC cell lines express ICN1 and ectopic ICN1 expression promotes xenograft tumor growth ${ }^{11,12}$. While pharmacological modulation of Notch paralogs represents an attractive strategy for cancer therapy ${ }^{13}$, a more detailed understanding of the functional role of the Notch pathway as it relates to tissue biology in the context of health and disease is necessary to guide such approaches.

In addition to squamous-cell differentiation, Notch1 regulates cell cycle $^{3}, 12,14$, senescence ${ }^{12}$, and epithelial-mesenchymal transition $(\mathrm{EMT})^{15-17}$. Acquisition of mesenchymal properties facilitates malignant transformation by limiting oncogeneinduced senescence ${ }^{18}, 19$. In human esophageal squamous cell carcinoma (ESCC), the deadliest form of all human SCCs ${ }^{20}$, EMT is associated with chemoresistance and poor prognosis ${ }^{21-23}$. EMT also regulates cancer stem cells (CSCs) ${ }^{24,25}$. CSCs defined by high CD44 expression $(\mathrm{CD} 44 \mathrm{H})$ have been identified in various tumor types, including SCCs ${ }^{26-29}$. In transformed esophageal and oral keratinocytes, cells with low CD44 expression (CD44L) and

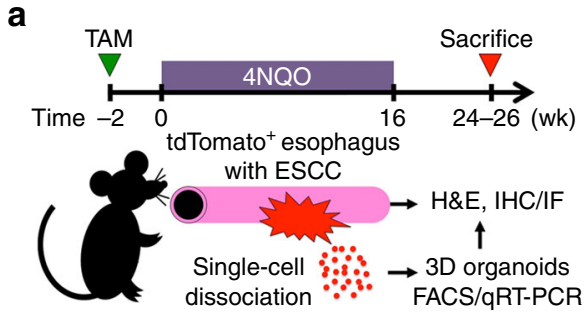

b
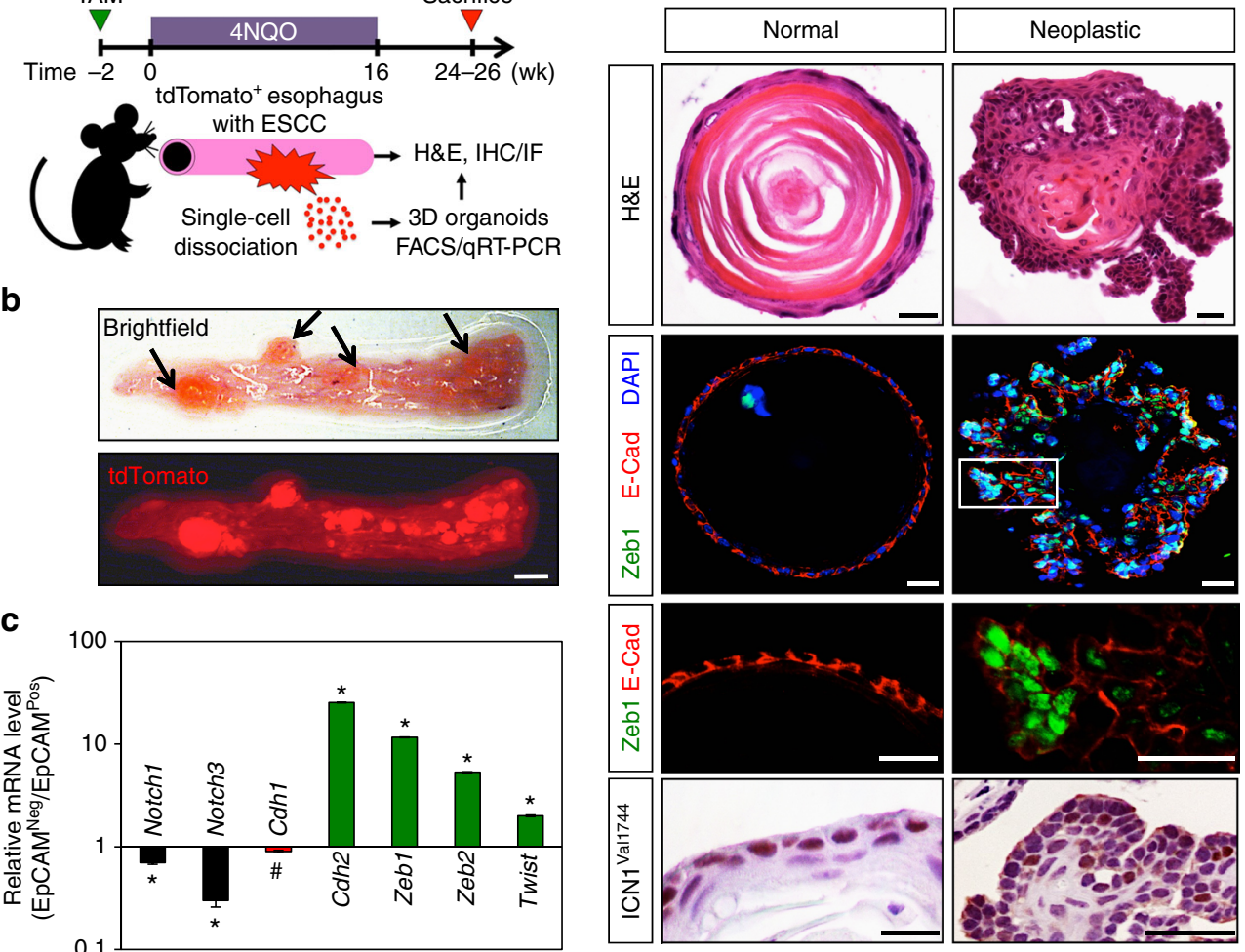

d

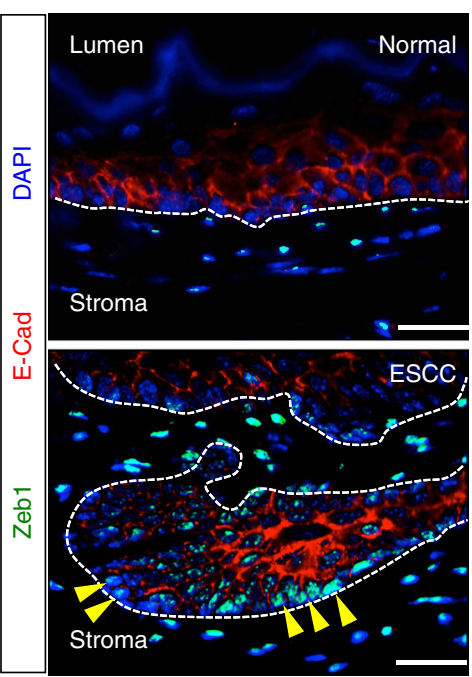

f

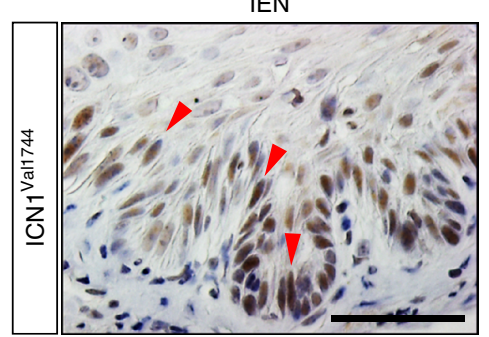

g

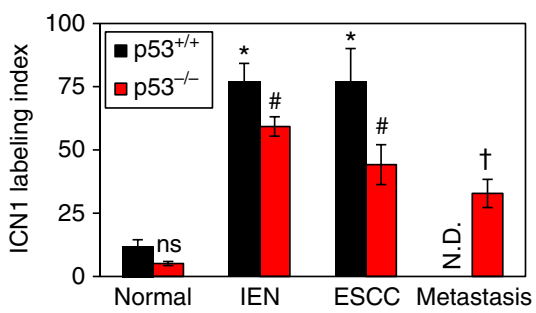


epithelial properties are converted to $\mathrm{CD} 44 \mathrm{H}$ cells with mesenchymal traits in response to transforming growth factor (TGF)- $\beta^{30}, 31$, a potent EMT inducer present in the tumor microenvironment ${ }^{32}$. During TGF $\beta$-mediated EMT, expression of the Notch ligand JAG1 is induced via $\mathrm{ZEB} 1^{12}, 15,33$, a transcription factor essential in TGF $\beta$-induced $\mathrm{EMT}^{34}, 35$ and microRNA-mediated regulation of Notch signaling ${ }^{33,36,37}$. While emerging lines of evidence support Notch1 as a positive effector of EMT ${ }^{15-17}, 37,38$, Notch3 limits the expansion of EMTcompetent esophageal keratinocytes ${ }^{11}$. Thus, although Notch1 and Notch3 cooperate to drive squamous-cell differentiation ${ }^{4}$, these Notch paralogs may play opposing roles in EMT and, potentially, regulation of CSC dynamics. The precise molecular mechanisms through which Notch signaling regulates distinct cell fates in a context-dependent manner have yet to fully elucidated.

Here, we aimed to define the functional role of Notch1 in SCC. We demonstrate that Notch1 activation and EMT are coupled to promote tumor initiation and intratumoral cancer cell heterogeneity in SCC. We find that the transcription factor ZEB1 represses $\mathrm{NOTCH} 3$, thereby limiting ICN1-induced differentiation while permitting ICN1-mediated EMT. Moreover, ICN1 expression in a small subset of SCC cells at the invasive tumor front predicts independently for poor prognosis of ESCC. These findings suggest an oncogenic role for Notch1 in SCC and identify the TGF $\beta$-ZEB1-Notch1 axis as potential target for SCC therapy.

\section{Results}

EMT and Notch1 activation are features of carcinogen-driven ESCC in vivo. To study SCC initiation and progression in vivo, we treated mice with 4-Nitroquinoline 1-oxide (4NQO), a potent oral-esophageal carcinogen. We combined $4 \mathrm{NQO}$ treatment with a cell-lineage tracing experimental system in which murine oral and esophageal epithelial basal cells (keratinocytes) were marked permanently with tdTomato fluorescent protein following tamoxifen (TAM)-induced Cre-mediated recombination in K5Cre ${ }^{E R T 2} ; R 26 t d T$ Tomato ${ }^{l s l / l s l}$ mice (Fig. 1a). 4NQO-induced lesions showed tdTomato accumulation (Fig. 1b; Supplementary Fig. 1a, b), validating the basal keratinocyte origin of these tumors $^{39}$. Flow cytometry revealed the presence of cells displaying both negative and positive expression of EpCAM (EpCAM ${ }^{\text {neg }}$ and EpCAM ${ }^{\text {pos }}$ ), an epithelial cell surface marker, within the tdTomato-positive (tdTomato ${ }^{\text {pos }}$ ) fractions of 4NQO-induced ESCC lesions (Supplementary Fig. 1c), suggesting a loss of epithelial characteristics in tumor cells originating from esophageal basal keratinocytes. In cell lineage tracing experiments, tdTomato expression assures that these EpCAM ${ }^{\text {neg }}$ cells are not co-existing intratumoral stromal cells (e.g., fibroblasts) which are not labeled with tdTomato via K5Cre $e^{E R T 2}$. Quantitative reverse-transcription polymerase chain reaction (qRT-PCR) analysis coupled with fluorescence-activated cell sorting (FACS) demonstrated further upregulation of genes associated with mesenchymal cells (Fig. 1c) including Zeb1, in tdTomato ${ }^{\text {pos }} ; \mathrm{EpCAM}^{\text {neg }}$ cells originating from basal esophageal keratinocytes. Consistent with EMT as described in human ESCC $^{11}$, neoplastic lesions displayed E-cadherin loss or mislocalization as well as robust Zeb1 expression at the stromal interface (Fig. 1d). Single-cell-derived three-dimensional (3D) organoids generated from 4NQO-induced dysplastic mucosa and primary tumors recapitulated acquisition of mesenchymal properties as found in 4NQO-induced neoplastic lesions (Fig. 1e). We also included mice with conditional loss or expression of mutant p53 since p53 dysfunction promotes EMT ${ }^{19,31,40}$ and found that more pronounced E-cadherin loss and reciprocal Zeb1 upregulation were observed in invasive tumors with mutant $\mathrm{p} 53^{\mathrm{R} 122 \mathrm{H}}$ (Supplementary Fig. 1d).

We next utilized this experimental platform to examine expression of ICN1 ( ICN1 $1^{\text {Vall744 }}$ ), the activated form of Notch1, throughout the natural history of ESCC. In comparison to normal esophageal squamous epithelia, ICN1 expression was increased in dysplastic lesions and remained upregulated in primary and metastatic ESCC (Fig. 1f, g; Supplementary Fig. 1e). Moreover, ICN1 was detected in invasive ESCC cells displaying E-cadherin mislocalization and Zeb1 upregulation (Supplementary Fig. 1d). ICN1 was also expressed highly in neoplastic organoids displaying EMT (Fig. 1e), suggesting a potential role for ICN1 in the acquisition of mesenchymal properties by SCC cells. The tumor suppressor p53 protein can transcriptionally activate Notch1 in response to genotoxic stress ${ }^{41}$. Since 4NQO activates $p 53$ via DNA damage ${ }^{42}$, ICN1 expression in 4NQO-induced early lesions may reflect p53 activation in dysplastic cells. Conversely, Notch1 downregulation may be accounted for by p53 inactivation during disease progression. Therefore, we evaluated the influence of p53 loss upon ICN1 expression in the esophageal epithelium. TAM-induced $K 5 C r e^{E R T 2}$-driven $p 53$ loss did not affect ICN1 expression in mice without 4NQO treatment $(P=0.06$ by Student's $t$-test; $n=3$ animals per group; two independent experimental replicates), a finding that recapitulates $\mathrm{p} 53^{-1-}$ murine epidermis ${ }^{43}$. When $p 53$ was deleted in oral-esophageal keratinocytes then mice were treated with 4NQO, $p 53$ loss did not prevent ICN1 expression in neither normal esophageal epithelium nor 4NQO-induced neoplastic lesions (Fig. 1g; Supplementary Fig. 1e). Mice with p53 deletion did, however, display frequent metastases (Supplementary Fig. 1a). These findings suggest that p53 may be dispensable for Notch1 activation in

Fig. 1 Lineage tracing reveals mesenchymal traits and Notch1 activation in ESCC lesions. a Schematic of cell-lineage tracing experiments. b Macroscopic and fluorescent images of representative tdTomato-labeled esophagus bearing 4 NQO-induced tumors (arrows). Scale bar, $1 \mathrm{~mm}$. c qRT-PCR analysis for indicated genes comparing EpCAM ${ }^{\text {neg }}$ to EpCAM ${ }^{\text {pos }}$ cells from a representative tdTomato-labeled tumor. ${ }^{\star} P<0.0001$ and ${ }^{\#} P<0.05$ vs. EpCAM ${ }^{\text {pos }}, n=3$. d Representative IF images for Zeb1 and E-cadherin (E-cad) in normal mucosa (top) and ESCC (bottom). Dashed line denotes interface between stroma and basal keratinocytes (top) or invasive ESCC cells (bottom). Note E-cadherin downregulation in ESCC cells with nuclear Zeb1 (arrowheads) at the tumor invasive front. Scale bar, $50 \mu \mathrm{m}$. e H\&E, multicolor IF for E-cadherin and Zeb1, and IHC for ICN1 (ICN1Val1744) in representative single-cell-derived organoids from normal mucosa or $4 \mathrm{NQO}$-induced tumors. Note that spherical organoids from 4NQO-untreated control mice exhibit a differentiation gradient with predominant luminal keratinization, whereas tumor-derived organoids (neoplastic) display irregular morphology with increased cellularity and diminished keratinization. Zeb1 expression was robust in tumor organoids, particularly at invasive protrusions with decreased E-cadherin expression and detectable ICN1 expression. Box denotes area magnified in panel below. Scale bars, $20 \mu \mathrm{m}$. f Representative IHC image for ICN1 4NQO-induced IEN (dysplasia) containing spindle-shaped cells (arrowheads). Scale bar, $50 \mu \mathrm{m}$. $\mathbf{g}$ Quantification of ICN1 IHC scoring in normal mucosa and 4NQO-induced

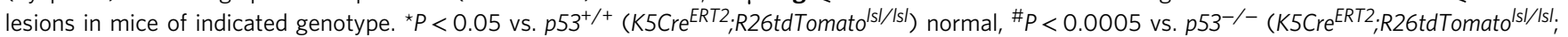
$\left.p 53^{\text {loxP/loxP }}\right)$ normal; ${ }^{\dagger} P<0.05$ vs. $p 53^{-/-}$IEN; ns not significant vs. $p 53^{+/+}$normal. Data in b-f represent at least three independent 4 NQO-induced lesions and $>20$ organoids from at least two independent experimental replicates. In $\mathbf{g}, n=5$ normal, $n=8$ IEN, and $n=5$ invasive ESCC in p53 ${ }^{+/+}$esophagi. N.D. not detectable. $n=3$ normal, $n=7$ IEN, $n=5$ invasive ESCC, and $n=4$ metastatic tumors in $p 53^{-/-}$esophagi. Two independent experimental replicates were carried out. Bar diagrams represent mean \pm s.d. in $\mathbf{c}$ and mean \pm s.e.m. in $\mathbf{g}$. Student's t-test was used for paired data comparisons in $\mathbf{c}$, g. ANOVA with Tukey's post hoc test was used for multiple comparisons in $\mathbf{g}$ 
a

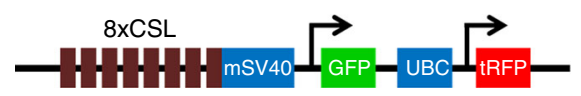

C

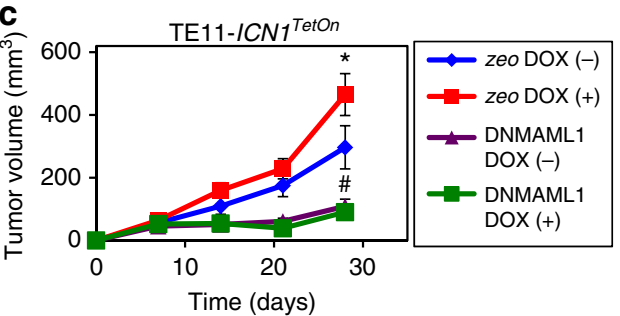

e

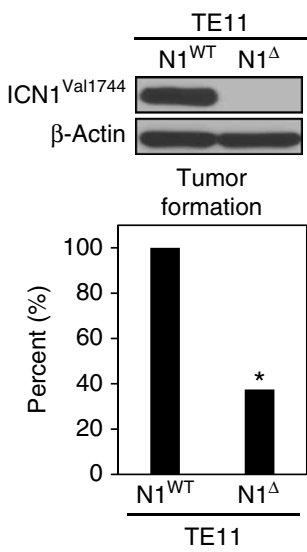

f b
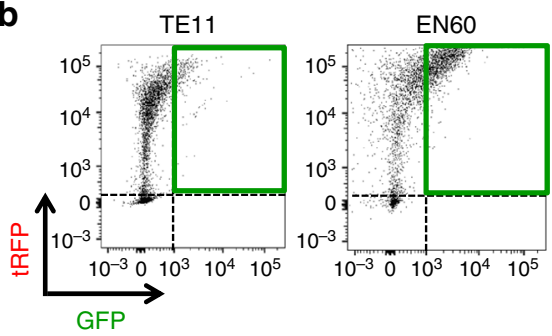

d
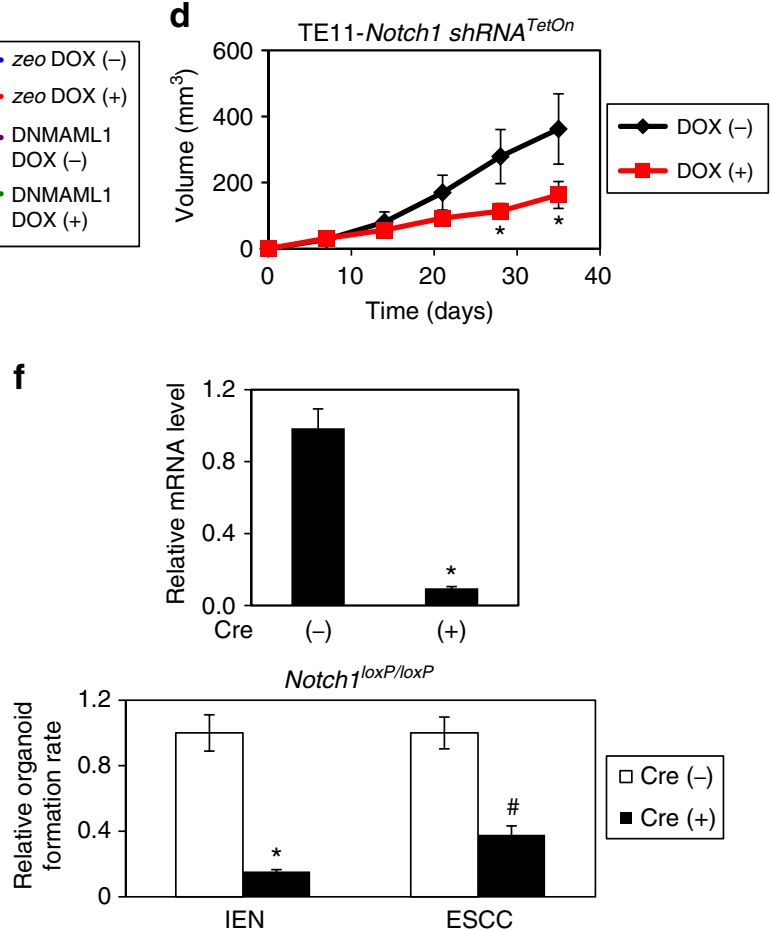

Fig. 2 Notch1 promotes ESCC tumorigenesis. a Schematic of 8xCSL-GFP reporter. Notch activation permits GFP reporter expression via concatemeric CSLbinding sites. Constitutively active UBC promoter drives tRFP expression concurrently. Cells without tRFP represent host-derived stromal cells. $\mathbf{b}$ Representative flow cytometry scatter plots determining $8 \times$ CSL-mediated GFP reporter activation, indicating Notch active population of tRFP-labeled ESCC cells in TE11 and EN60 xenograft tumors. On average, $43.5 \pm 0.5 \%$ s.d. of EN60 and $11.2 \pm 6.5 \%$ s.d. of TE11 cells comprised the live GFPPos/tRFPPos fraction across three independent tumors. $\mathbf{c}$, $\mathbf{d}$ Tumor growth curves in immunodeficient mice carrying TE11 tumors of indicated genotypes. Upon xenograft transplantation, mice were treated with DOX to induce ICN1 or NOTCH1-directed shRNA. Tumor growth was monitored for indicated time periods. In $\mathbf{c}$, ${ }^{\star} P$ $<0.01$ vs. zeo and DOX $(-),{ }^{\#} P<0.05$ vs. zeo and DOX $(+), n=6-8$ per group. In $\mathbf{d},{ }^{\star} P<0.05$ vs. DOX $(-) ; n=6$ per group. e TE11 cells with CRISPR/ Cas9-mediated NOTCH1 deletion. Immunoblotting confirms ICN1 loss in NOTCH1-deleted TE11 $\left(\mathrm{N1}^{\Delta}\right)$, but not non-targeted control TE11 (N1 ${ }^{\mathrm{WT}}$ ) cells. Bar graph shows tumor formation rate in immunodeficient mice 7 weeks after xenograft transplantation. ${ }^{\star} P<0.05$ vs. N1WT, $n=8$ per group. $\mathbf{f}$ Esophageal keratinocytes isolated from $4 \mathrm{NQO}$-induced IEN (dysplasia) lesions or ESCC tumors of Notch7 ${ }^{\text {loxP/loxP }}$ mice were utilized to generate 3D esophageal organoids in the presence or absence of ex vivo Cre-mediated recombination. qRT-PCR analysis confirms inhibition of Notch1 gene expression upon ex vivo Cre-mediated recombination in ESCC organoids. ${ }^{\star} P<0.000$ vs. Cre $(-), n=3$. Organoid formation rate was evaluated at day 14 post-plating and is represented as relative to $\mathrm{Cre}(-)$ for IEN and ESCC. ${ }^{\star} P<0.000$ vs. Cre $(-)$ IEN; ${ }^{\#} P<0.001$ vs. Cre (-) ESCC. Line graphs represent mean \pm s.e.m. in $\mathbf{c}$, d. Bar diagrams represent mean \pm s.d. in $\mathbf{f}$. At least two independent replicates were performed for all experiments. Repeated measures ANOVA with Tukey's post hoc test were used for multiple comparisons in c, d. Chi square with Fisher's exact test was used for percentage comparisons in e. Student's t-test was used for paired data comparisons in $\mathbf{f}$

4NQO-induced lesions, albeit essential for overall SCC progression.

Notch1 promotes ESCC tumorigenicity and expansion of CD44H cells with mesenchymal properties. To explore further the functional role of Notch1 in ESCC tumorigenicity, we first utilized the extensively characterized human ESCC cell lines TE11 and EN60 ${ }^{12}$. Both express ICN1 and form tumors upon xenograft transplantation in immunodeficient mice where doxycycline (DOX)-inducible ectopic ICN1 augments tumor growth ${ }^{11,12}$. To assess Notch activity in vivo, we used $8 \times C S L-G F P$, a lentiviral green fluorescent protein (GFP) reporter driven by the minimal
SV40 promoter fused to concatemeric CSL-binding sites (Fig. 2a). In TE11 and EN60 xenograft tumors, a subset of turboRFP (tRFP)-expressing tumor cells exhibited CSL-mediated transcriptional activity (Fig. 2b). A functional role for Notch activity in ESCC tumor growth is evident as dominant negative mutant MAML1 (DNMAML1) ${ }^{44}$ prevented DOX-induced ectopic ICN1 from stimulating tumor growth (Fig. 2c). Additionally, DNMAML1 alone or NOTCH1-directed short hairpin RNA (shRNA) suppressed tumor volume significantly (Fig. 2c, d). CRISPR/Cas9-mediated NOTCH1 deletion dramatically impaired tumor formation by TE11 cells in immunodeficient mice (Fig. 2e), supporting a role for Notch1 in tumor initiation as well. 
a

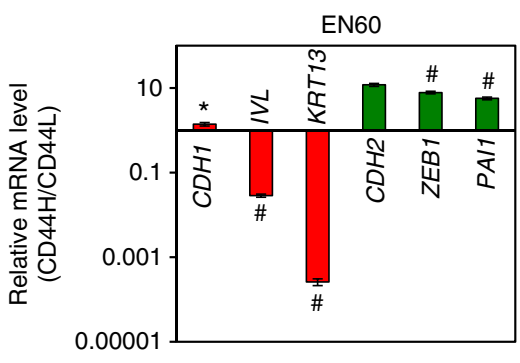

C
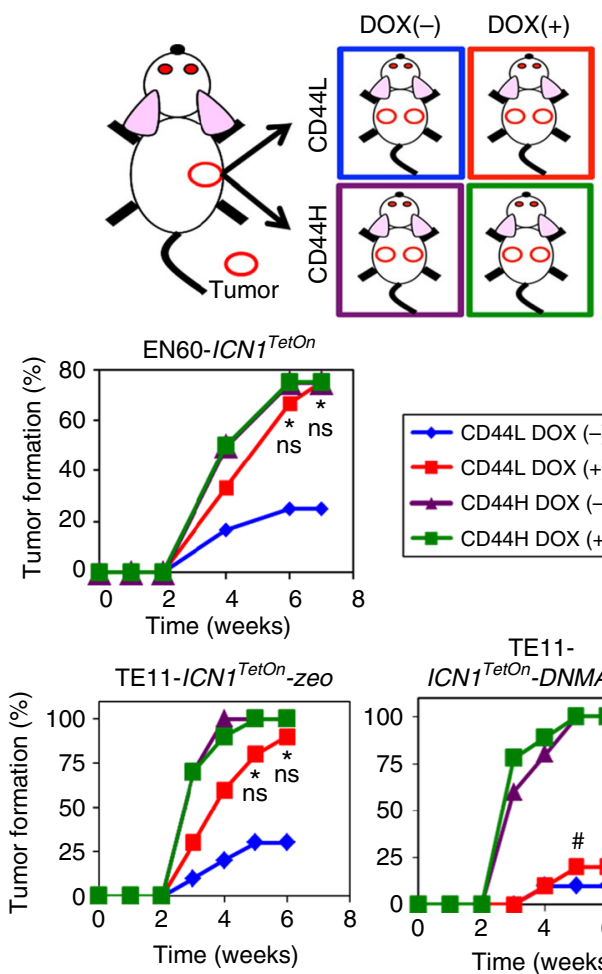

b

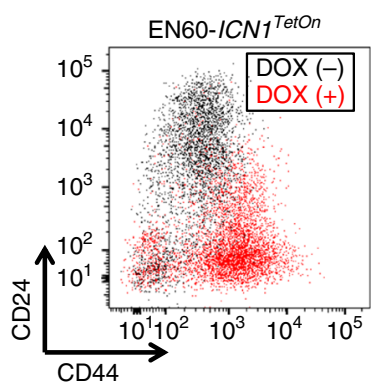

d

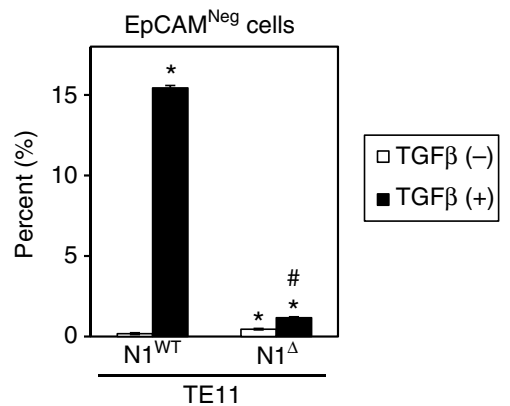

e

Cre $\quad(-)$

$(+)$

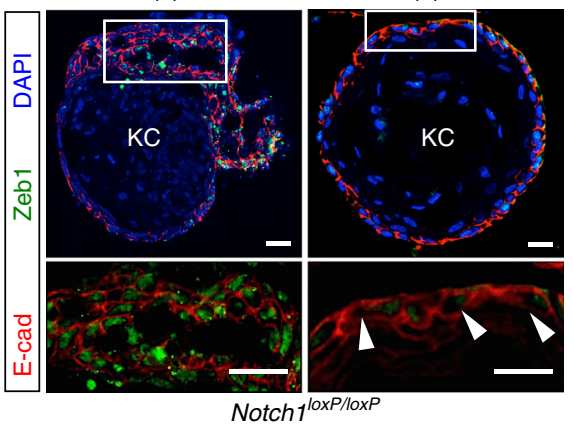

Fig. 3 Notch1 facilitates expansion of CD44H cells with mesenchymal properties. a qRT-PCR analysis for indicated genes comparing EN60 intratumoral CD44L and CD44H cells. ${ }^{\star} P<0.05$ vs. CD44L; ${ }^{\#} P<0.0001$ vs. CD44L; $n=3$ per group. b Representative flow cytometry scatter plot determining CD44H cells in EN60 tumors grown for 4 weeks with or without DOX-induced ICN1 (EN60-ICN1 TetOn). c Experimental design of serial transplantation experiments and tumor formation rates by CD44L and CD44H cells purified from parental xenograft tumors carrying DOX-inducible ICN1 (EN60-ICN1 TetOn and TE11$I C N 7^{\text {TetOn }}$ ). Parental tumors were grown in mice without DOX treatment and dissociated for FACS-purification of CD44L and CD44H cells. Purified cells were serially transplanted $\left(10^{3}\right.$ cells per injection site) into recipient mice to monitor tumor formation. Recipient mice were treated with or without DOX. TE11 carried DNMAML1 or zeo (empty vector control). ${ }^{\star} P<0.05$ for CD44L and DOX (+) vs. CD44L and DOX $(-)$, $n=10-12$ per group; ns not significant for CD44L and DOX (+) vs. CD44H (with or without DOX treatment), $n=10$ per group; ${ }^{\#} P<0.05$ vs. TE11-ICN1 ${ }^{\text {TetOn }}$-zeo CD44L and DOX (+), $n=10$ per group. d Flow cytometry analysis for EpCAM-negative cells in TE11 with NOTCH1 deletion $\left(\mathrm{N} 1^{\Delta}\right)$ or wild-type control $\left(\mathrm{N} 1^{\mathrm{WT}}\right)$. Cells were treated with or without $5 \mathrm{ng} / \mathrm{ml} \mathrm{TGF} \beta$ for $72 \mathrm{~h} .{ }^{\star} P<0.05$ vs. TGF $\beta(-) ;{ }^{\#} P<0.0001$ vs. N1 ${ }^{\mathrm{WT}}$ and TGF $\beta(+), n=3$. Data are presented as mean \pm s.e.m. e Multicolor IF for E-cadherin and Zeb1 in representative single-cell-derived organoids from 4 NQO-induced ESCC tumors of Notch $7^{\text {loxP/loxP }}$ mice with or without ex vivo Cremediated recombination. Zeb1 expression diminished in organoids upon Cre-mediated Notch1 deletion (arrowheads). Box denotes area that is magnified in panel below. KC keratinized core of organoids. Scale bars, $20 \mu \mathrm{m}$. Bar diagrams represent mean \pm s.d. in a, d. At least two independent replicates were performed for all experiments. Student's $t$-test was used for paired data comparisons in $\mathbf{a}$, $\mathbf{d}$. Chi square with Fisher's exact test was used for percentage comparisons in $\mathbf{c}$

EN60 cells are less tumorigenic than TE11 upon xenograft transplantation, displaying $12.5-25 \%$ tumor formation rates with $1-4 \times 10^{6}$ cells transplanted; however, DOX-induced ectopic ICN1 enhanced EN60 tumor formation rate to $100 \%(P<0.01$ by Fisher's exact test; $n=12$; two independent experimental replicates). Moreover, the ability of esophageal neoplastic cells to form single-cell-derived 3D organoids was attenuated upon Cremediated ex vivo Notch1 deletion in single-cell suspensions prepared from dysplastic lesions or ESCC tumors of 4NQOtreated Notch $1^{\text {loxP/loxp }}$ mice (Fig. 2f).

Human SCC tumors comprise CD44L and CD44H cells, the latter exhibiting augmented tumor-initiating capability ${ }^{28,} 29$. $\mathrm{CD} 44 \mathrm{H}$ expression is associated with mesenchymal characteristics in transformed oral-esophageal cell lines ${ }^{31}$. Following FACS-purification, early passage CD44H TE11 and EN60 cells maintained enhanced expression of the mesenchymal cell marker 
a
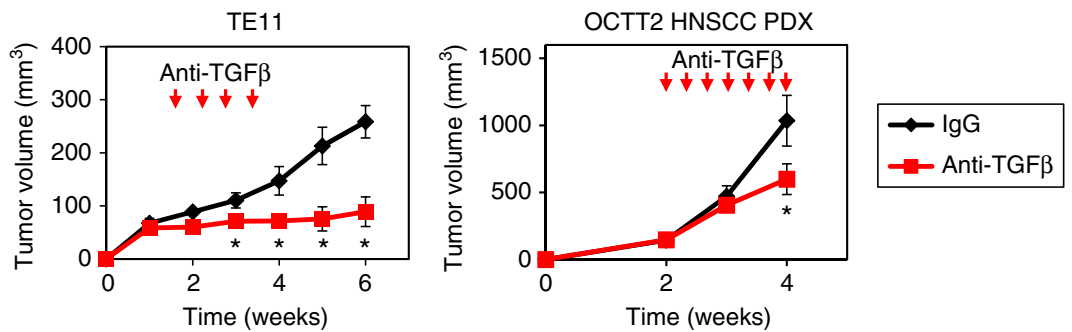

b
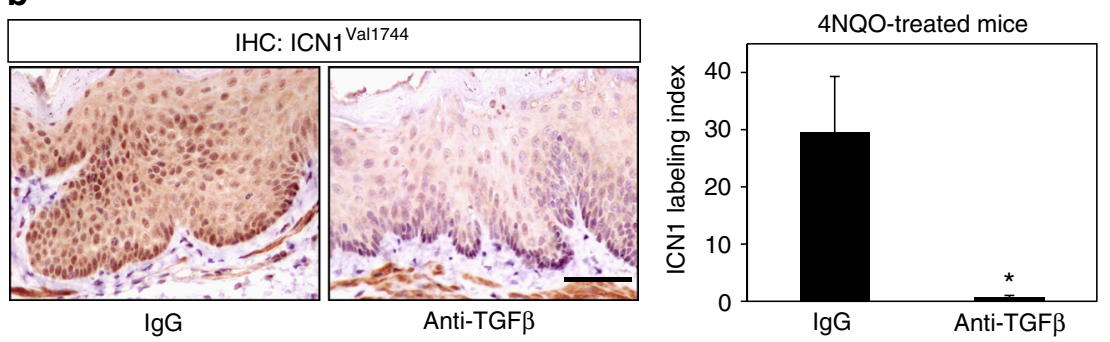

C

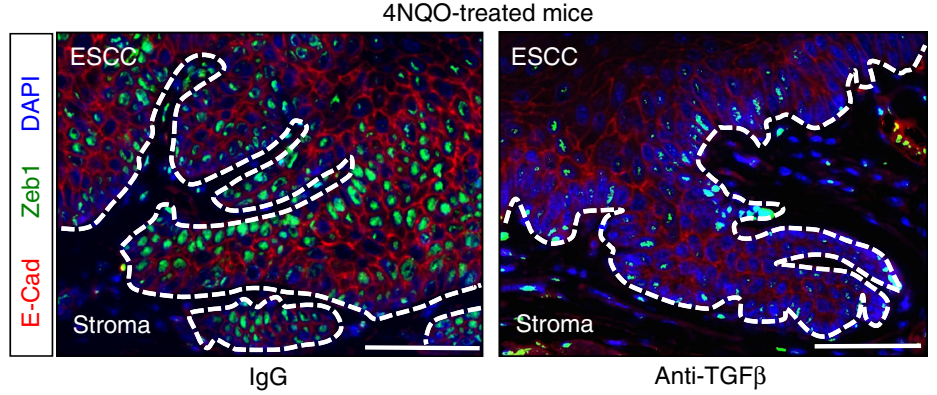

Fig. 4 TGF $\beta$ signaling facilitates ESCC tumor growth, Notch1 signaling, and EMT. a Growth curve for mice bearing TE11 or HNSCC PDX tumors receiving either ID11 anti-TGF $\beta$ blocking antibody or control IgG via intraperitoneal injection at indicated time points (arrows). ID11 neutralizes all TGF $\beta$ isoforms. ${ }^{\star} P$ $<0.05$ vs. IgG, $n=5-8$ per group. b, c $p 53^{+/+}$mice were treated with $4 \mathrm{NQO}$ for 16 weeks. Six weeks following $4 \mathrm{NQO}$ withdrawal, mice were treated with ID11 anti-TGF $\beta$ blocking antibody or control IgG via intraperitoneal injection three times weekly for a period of 2 weeks. In $\mathbf{b}$, quantification of ICN1 IHC scoring in esophageal epithelium with representative IHC images for lgG-treated and anti-TGF $\beta$ blocking antibody-treated animals. ${ }^{\star} P<0.05 ; n=4-5$ per group. Scale bar, $50 \mu \mathrm{m}$. In c, multicolor IF for E-cadherin and Zeb1 in representative 4NQO-induced IEN lesions from IgG-treated and anti-TGF $\beta$ blocking antibody-treated animals. Data indicate mean \pm s.e.m. in $\mathbf{a}, \mathbf{b}$. At least two independent replicates were performed for all experiments. Repeated measures ANOVA with Tukey's post hoc test were used for multiple comparisons in $\mathbf{a}$. Student's $t$-test was used for paired data comparisons in $\mathbf{b}$

$\mathrm{N}$-cadherin as compared to their CD44L counterparts in culture (Supplementary Fig. 2a). Additionally, CD44H cells isolated from TE11 and EN60 xenograft tumors exhibited upregulation of ZEB1 and $\mathrm{CDH} 2$ (N-cadherin) (Fig. 3a; Supplementary Fig. 2b). As ectopic ICN1 expression in EN60 tumors enhanced robustly intratumoral CD44H cell content (Fig. 3b), we hypothesized that Notch1 contributes to tumor initiation via generation of $\mathrm{CD} 44 \mathrm{H}$ cells that have mesenchymal properties. To determine how Notch1 may influence tumor initiation by CD44L and CD44H cells, we performed serial transplantation experiments (Fig. 3c). We first grew tumors without activating DOX-inducible ICN1. We then purified CD44L and CD44H cells by FACS from primary tumors and $1 \times 10^{3}$ cells were serially injected into recipient mice where DOX was given to induce ectopic ICN1 expression. CD44L cells purified from EN60 and TE11 tumors showed low $(<30 \%)$ spontaneous tumor formation efficiency; however, ICN1 dramatically stimulated tumor initiation by CD44L cells to $80-90 \%$. In TE11 CD44L cells, DNMAML1 not only antagonized ICN1-mediated tumorigenicity, but also suppressed spontaneous tumor formation. While purified CD44H cells were highly tumorigenic (80-100\%), neither ectopic ICN1 nor DNMAML1 affected tumor initiation by CD44H cells, suggesting that established $\mathrm{CD} 44 \mathrm{H}$-mediated tumor initiation is independent of Notch1. To our knowledge, this is the first demonstration that Notch1 may facilitate tumor initiation by converting CD44L cells to highly tumorigenic $\mathrm{CD} 44 \mathrm{H}$ cells with mesenchymal traits in vivo.

TGF $\beta$ and Notch1 cooperate to drive EMT in the tumor microenvironment. The functional role of Notch1 in EMT was suggested as NOTCH1 deletion in cultured TE11 cells attenuated sharply TGF $\beta$-mediated EpCAM ${ }^{\text {neg }}$ cell induction (Fig. 3d). Cremediated ex vivo Notch1 deletion in 3D ESCC organoids generated from 4NQO-induced Notch $1^{\text {loxP/loxP }}$ murine tumors resulted in a diminished expression of Zeb1 (Fig. 3e). Moreover, DOXinduced ectopic ICN1 augmented intratumoral $\mathrm{EpCAM}^{\text {neg }}$ cell content in TE11 xenograft tumors (Supplementary Fig. 2c). While these data support a role for Notch1 in promotion of ESCC tumor cells with attributes of EMT, EpCAM ${ }^{\text {neg }}$ cells were rare in cultured TE11 cells and ectopic ICN1 expression alone had no influence upon this cell population (Supplementary Fig. 2c), indicating that Notchl activation alone may not be sufficient to drive EMT.

In the tumor microenvironment, Notch signaling may be modulated by other transcription factors such as HIF $1 \alpha^{17}$ and 
SMAD ${ }^{45}$ via physical interactions with ICN1. We suspected that TGF $\beta$ influences Notch1-mediated tumor promotion and EMT since the TGF $\beta$ target gene PAI1 was upregulated in CD44H cells in tumors (Fig. 3a; Supplementary Fig. 2b). In agreement, an antiTGF $\beta$ blocking therapeutic monoclonal antibody $1 \mathrm{D} 11^{46}$ severely impaired tumor growth of TE11 xenograft tumors as well as OCTT2 head and neck SCC patient-derived xenografts (PDXs)
(Fig. 4a). In the context of 4NQO-mediated carcinogenesis, the percentage of esophageal mucosa occupied by neoplastic lesions classified as intraepithelial neoplasia (IEN) or ESCC was diminished upon treatment with $1 \mathrm{D} 11(26.0 \% \pm 8.1$ s.e.m.; $n=$ 5) as compared to isotype control $(56.3 \% \pm 14.2$ s.e.m.; $n=4 ; P=$ 0.09 by Student's $t$-test; two independent experimental replicates). Administration of $1 \mathrm{D} 11$ to $4 \mathrm{NQO}$-treated animals further

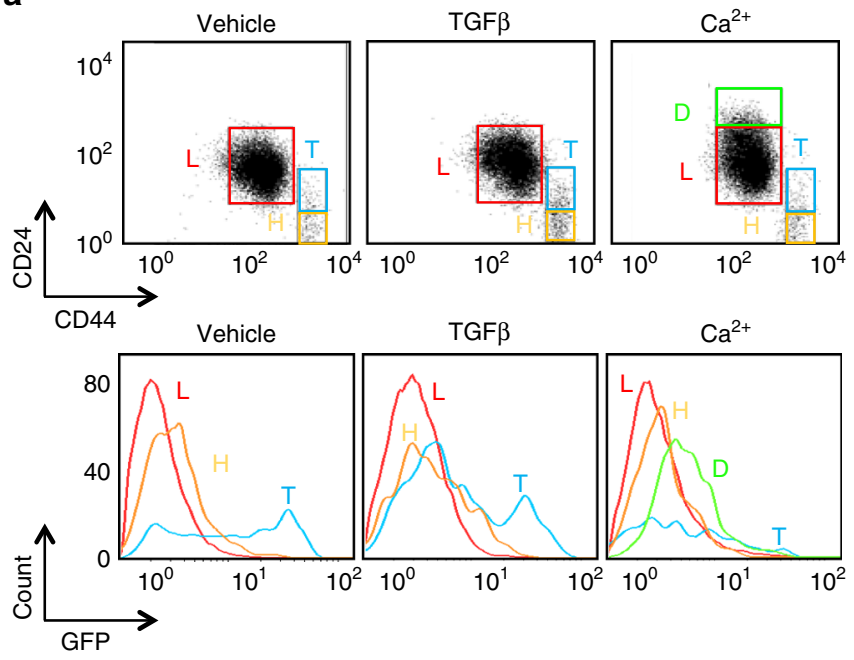

b
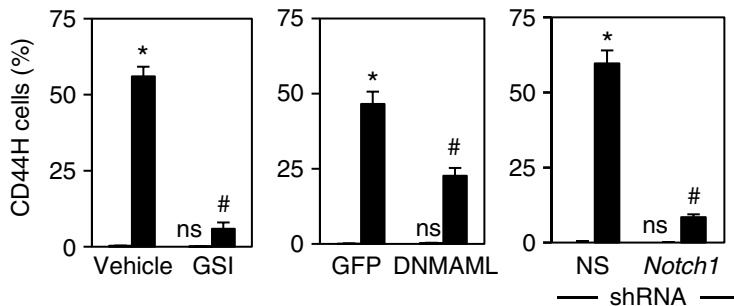

C

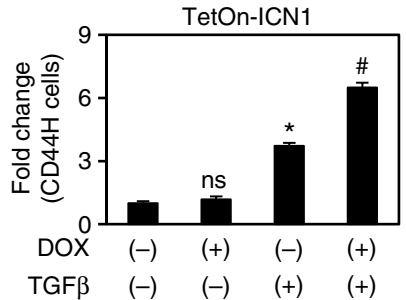

d

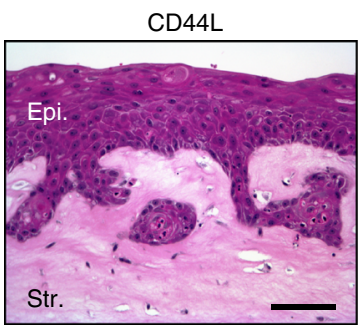

$\mathrm{CD} 44 \mathrm{H}$

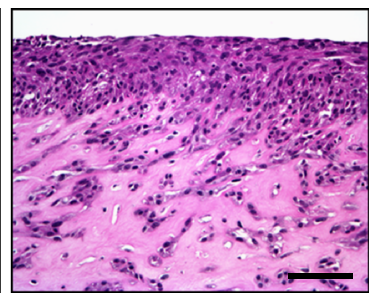

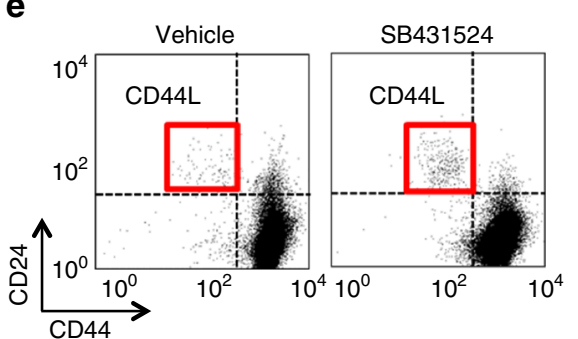

$\mathbf{f}$

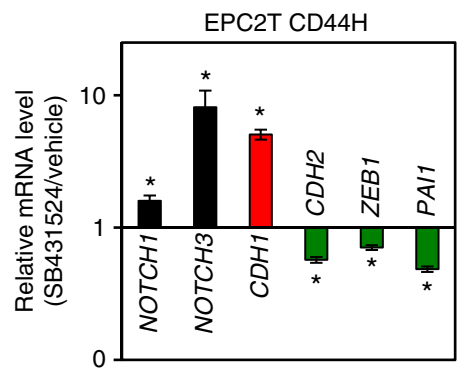

g

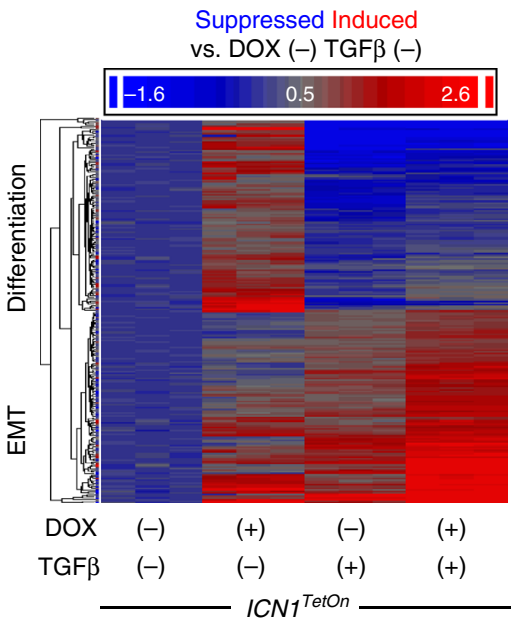

h

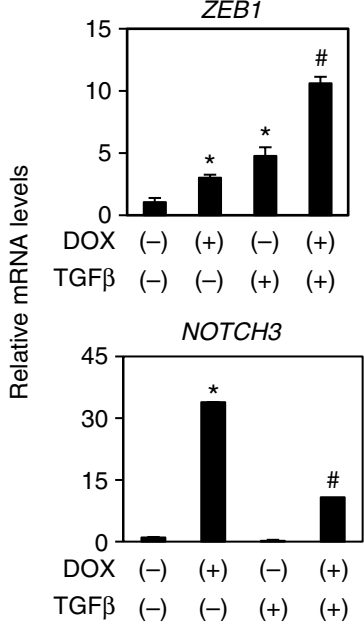

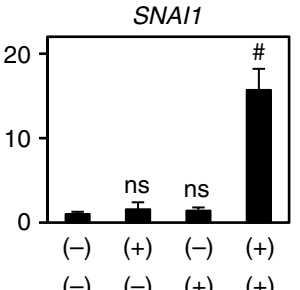

$(-) \quad(-) \quad(+) \quad(+)$

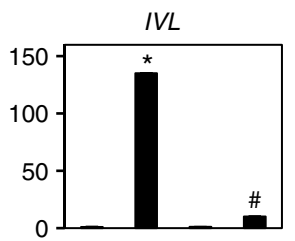

$(-) \quad(+) \quad(-) \quad(+)$

$(-) \quad(-) \quad(+) \quad(+)$ 
attenuated ICN1 expression as well as morphological evidence of EMT in esophageal epithelium (Fig. 4b, c). Taken together, these data indicate that TGF $\beta$ functions as a critical positive effector of Notch1 and EMT in the context of the tumor microenvironment.

To dissect further the mechanistic role of Notch1 in the generation and maintenance of ESCC tumor cells with mesenchymal features, we utilized the genetically engineered transformed esophageal cell line EPC2T (EPC2-hTERT-EGFR-p53 ${ }^{\mathrm{R} 175 \mathrm{H}_{-}}$ cyclin D1), comprising discrete CD44L and CD44H subpopulations with epithelial and mesenchymal traits, respectively ${ }^{31}$ (Supplementary Fig. 3a). Under basal conditions, Notch activity, as measured using $8 \times C S L-G F P$ reporter (Fig. 2a), was highest within a subset of intermediate transitioning cells (designated as CD44T) as compared to CD44L or CD44H cells (Fig. 5a). In TGF $\beta$-mediated EMT with a resulting increase in CD44T and $\mathrm{CD} 44 \mathrm{H}$ cells, Notch activity was augmented further in the CD44T subpopulation (Fig. 5a), indicating that Notch activity may be augmented transiently during the CD44L-to-CD44H transition. Induction of $\mathrm{CD} 44 \mathrm{H}$ cells may be accounted for by expansion of pre-existing CD44H cells as well as conversion from CD44L cells. Following FACS purification, $\mathrm{CD} 44 \mathrm{H}$ cells failed to display CD44L repopulation even after extended passage (Supplementary Fig. 3b). CD44L cells that were cultured in the absence of TGF $\beta$ underwent expansion while permitting minimal $\mathrm{CD} 44 \mathrm{H}$ cell repopulation within 5 weeks (four passages) (Supplementary Fig. 3b); however, purified CD44L cells robustly gave rise to CD $44 \mathrm{H}$ cells upon TGF $\beta$ stimulation (Supplementary Fig. 3c; Fig. 5b). A requirement for TGF $\beta$ in $\mathrm{CD} 44 \mathrm{H}$ cell induction was suggested as pharmacological inhibition of TGF $\beta$ receptormediated signaling suppressed spontaneous conversion of purified CD44L cells to CD44H cells (Supplementary Fig. 3d). A permissive role for Notch1 in $\mathrm{CD} 44 \mathrm{H}$ cell generation via TGF $\beta$-induced EMT was implicated further as a $\gamma$-secretase inhibitor (GSI), DNMAML1 or NOTCH1-directed shRNA each individually attenuated TGF $\beta$-mediated $\mathrm{CD} 44 \mathrm{H}$ cell expansion from purified CD44L cells (Supplementary Fig. 3c; Fig. 5b). Ectopic ICN1 augmented CD44H cell expansion from TGF $\beta$ stimulated purified $\mathrm{CD} 44 \mathrm{~L}$ cells where ICN1 failed to influence spontaneous $\mathrm{CD} 44 \mathrm{H}$ cell expansion in the absence of TGF $\beta$ (Fig. 5c). Taken together, these data indicate that Notch1 activation is required, but not sufficient, for EMT-mediated $\mathrm{CD} 44 \mathrm{H}$ cell induction in EPC2T cells.

Transcriptional repression of Notch3 via Zeb1 permits Notch1mediated EMT. As our findings indicate a role for Notch in EMT, we next sought to determine how Notch1 regulates cell fate in squamous epithelia. The $8 \times C S L-G F P$ reporter activity was unaffected in CD44T and CD44H cells upon calcium-mediated squamous-cell differentiation (Fig. 5a) ${ }^{4}$. In CD44L cells, which displayed differentiation in organotypic 3D culture (OTC) when compared to invasive $\mathrm{CD} 44 \mathrm{H}$ cells (Fig. 5d), calcium treatment enhanced Notch activity in a subset of cells (Fig. 5a). Thus, local environmental cues may impact Notch signaling to direct cell fate determination. In agreement with this notion, pharmacological inhibition of TGF $\beta$ signaling in purified CD $44 \mathrm{H}$ cells induced CD44L cells with concurrent downregulation of EMT markers and $\mathrm{NOTCH} 3$ upregulation (Fig. 5e, f). A trend toward increased Notch 3 expression was also detected in peeled murine esophageal epithelia with 4NQO-induced neoplastic lesions following treatment with anti-TGF $\beta$-blocking antibody 1D11 (Supplementary Fig. 3e). Gene array analysis in EPC2T cells revealed further that ectopic ICN1 expression induced a gene expression pattern compatible with squamous-cell differentiation. However, TGF $\beta$ treatment in the context of ICN1-overexpression triggered a robust shift toward an EMT-associated gene expression signature (Fig. 5g; Supplementary Fig. 3f) (GSE37994, GSE37993). qRTPCR analysis confirmed that ICN1 and TGF $\beta$ cooperate to induce expression of EMT regulators, including ZEB1 and SNAI1, while TGF $\beta$ limited ICN1-mediated expression of NOTCH3 as well as $I V L$, the latter a marker of terminal differentiation (Fig. 5h). Although the lack of discontinuous CD44L and CD44H cell populations in EN60 and TE11 prevented their isolation for longterm cell culture analyses, the role of Notch and TGF $\beta$ signaling in induction of $\mathrm{CD} 44 \mathrm{H}$ cells and regulation of squamous-cell differentiation was recapitulated in TE11 cells (Supplementary Fig. 3g-i).

We investigated next the mechanistic role of Notch3 in EMT in the context of ESCC. TE11 serial transplantation experiments revealed that DOX-induced ectopic ICN3 suppressed tumor initiation by CD44L cells (Fig. 6a). In EPC2T cells, ectopic ICN3 induced IVL in the presence or absence of TGF $\beta$ stimulation (Supplementary Fig. 4a, b). Moreover, ectopic ICN3 abrogated TGF $\beta$-mediated CD44H cell expansion in EPC2T cells (Supplementary Fig. 4c), while NOTCH3 knockdown was sufficient to promote $\mathrm{CD} 44 \mathrm{H}$ cell expansion (Fig. 6b) coupled with decreased differentiation and increased EMT characteristics (Fig. 6c) in the absence of TGF $\beta$ stimulation. These findings indicate that Notch3, unlike Notch1, may limit EMT so as to permit squamous-cell differentiation. To define the mechanism through which Notch3 expression is suppressed during EMT, we analyzed the NOTCH3 locus by the ECR browser ${ }^{47}$. This analysis predicts two conserved ZEB-binding sites in the $\mathrm{NOTCH} 3$ second intron (N3Int2) adjacent to the CSL-binding sites (Fig. 6d) that is occupied by ICN1 during squamous-cell differentiation ${ }^{4}$. Hypothesizing that ZEB transcription factors repress Notch3, we evaluated the influence of ectopic ZEB1 or

Fig. 5 TGF $\beta$-mediated Notch1 activation permits EMT and suppresses differentiation. a Representative flow cytometry scatter and histogram plots for $8 \times C S L-G F P$ reporter activity in indicated subpopulations of EPC2T cells treated with TGF $\beta$ or $\mathrm{CaCl}_{2}$ for $72 \mathrm{~h}$. b, $\mathbf{c}$ Flow cytometry determined CD44H cells induced in FACS-purified CD44L cells with indicated conditions or genotypes. Cells were treated with TGF $\beta$ or DOX for 7 days. ${ }^{\star} P<0.0001$ vs. TGF $\beta(-)$; ${ }^{\#} P<0.005$ vs. TGF $\beta(+)$ and DMSO, GFP, or non-silencing scrambled (NS) shRNA in $\mathbf{b} ; n=3$. ${ }^{\star} P<0.05$ vs. TGF $\beta(-)$ and DOX (-); ns not significant vs. TGF $\beta(-)$ and DOX (-); ${ }^{\#} P<0.05$ vs. TGF $\beta(+)$ and DOX (-) in c; $n=3$. d Representative H\&E images of OTC reconstituted with CD44L or CD44H cells. Epi epithelia, Str stroma. Scale bar, $50 \mu \mathrm{m}$. e Representative flow cytometry scatter plots of purified CD44H cells treated with SB431542 or vehicle (control) for 14 days. SB431542 increased CD44L cell content (upper left quadrant) to $0.35 \pm 0.08 \%$ as compared to $1.2 \pm 0.1 \%$ s.d. in vehicle $(P<$ $0.0005, n=3)$. $\mathbf{f}$ qRT-PCR analysis for indicated genes in FACS-purified CD44H cells treated with or without SB431542. mRNA level for each gene in vehicle-treated cells was set as $1 .{ }^{\star} P<0.0005$ vs. vehicle, $n=3$. $\mathbf{g}$ Heat map of gene array results from EPC2T derivative (EPC2T-ICN1 ${ }^{T e t O n}$ ) treated with DOX and/or TGF $\beta$ ( $n=3$ per condition). "Differentiation" genes were significantly increased ( $\geq 1.5$-fold) by DOX-induced ectopic ICN1 alone, but suppressed by TGF $\beta$ ( $P<0.05$ by two-way ANOVA). "EMT" genes activated by TGF $\beta$ ( $\geq 1.5$-fold) were significantly augmented by ectopic ICN1 ( $P<0.05$ by two-way ANOVA). $\mathbf{h}$ qRT-PCR analysis validates gene array results in $\mathbf{g}$. mRNA level for DOX $(-)$ and TGF $\beta(-)$ was set to 1 . ${ }^{\star} P<0.001$ vs. TGF $\beta(-)$ and DOX $(-) ;{ }^{\#} P<0.0001$ vs. TGF $\beta(-)$ and DOX $(+)$; ns not significant vs. TGF $\beta(-)$ and DOX $(-) ; n=3$. All bar diagrams indicate mean \pm s.d. Student's $t-$ test was used for paired data comparisons in $\mathbf{e}, \mathbf{f}$. At least two independent replicates were performed for all experiments. ANOVA with Tukey's post hoc test were used for multiple comparisons in $\mathbf{b}, \mathbf{c}, \mathbf{h}$ 
a

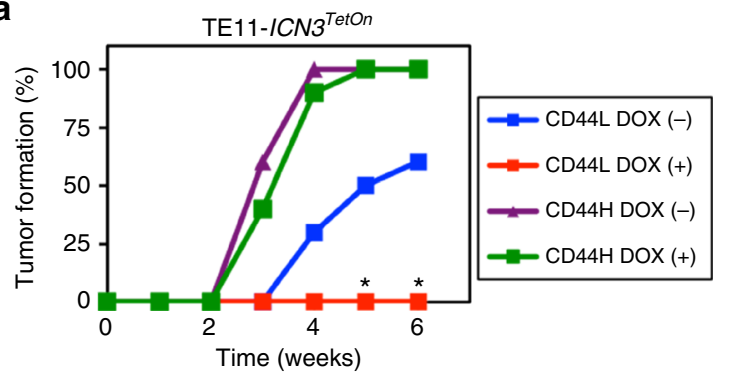

b

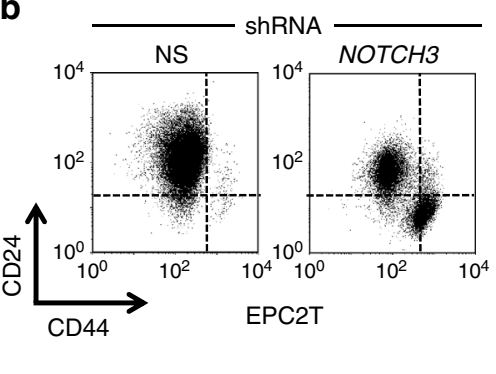

C

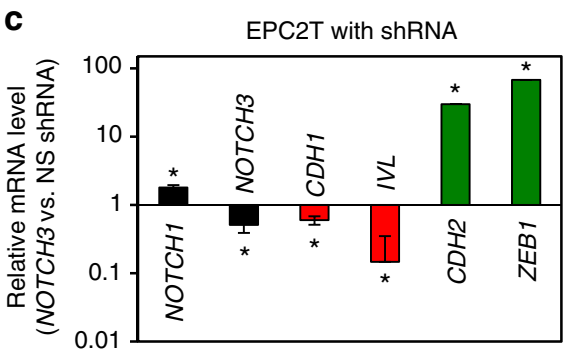

d $\mathrm{NOTCH3}$

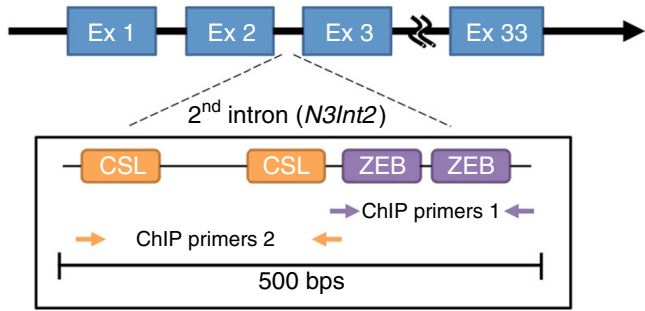

e

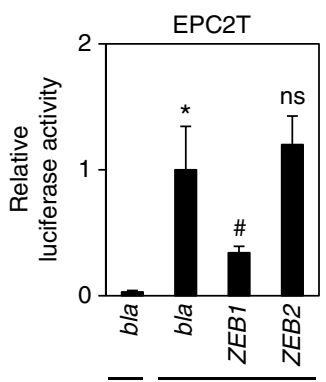

f

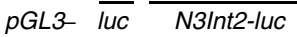
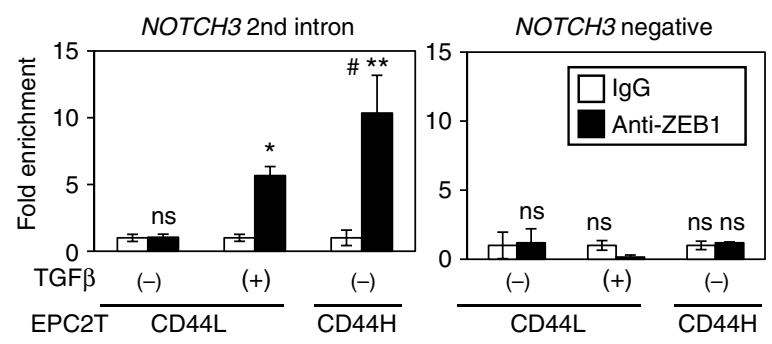

g
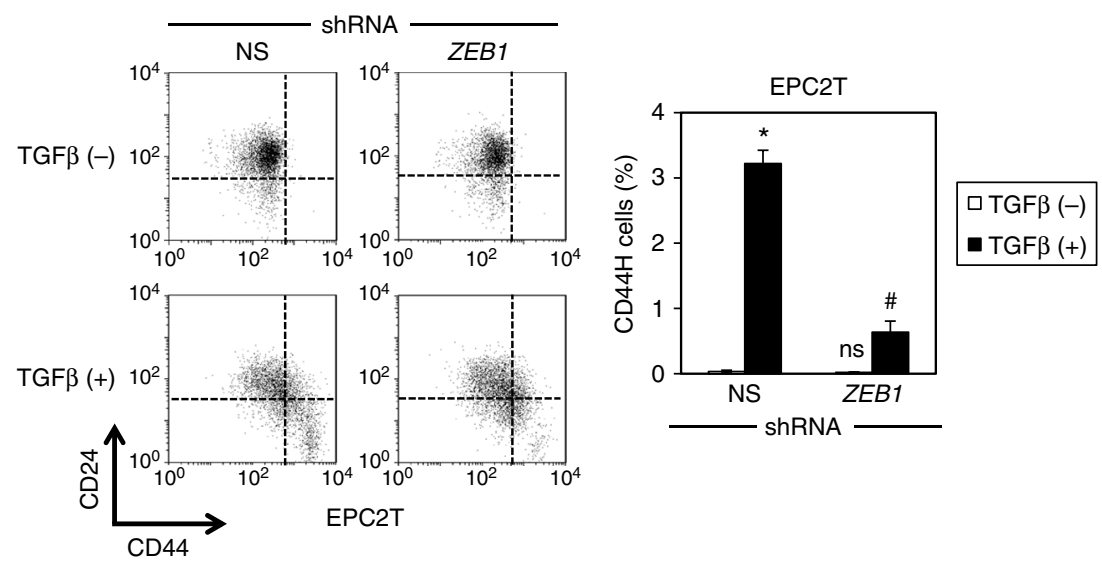

Fig. 6 ZEB1 represses NOTCH3, facilitating EMT and tumor initiation. a Serial transplantation experiments with DOX-inducible ICN3I-expressing TE11 (TE11$\left.I_{\text {ICN3 }}{ }^{\text {TetOn }}\right)$. Tumor formation rates were determined as in Fig. 2e. ${ }^{\star} P<0.05$ vs. CD44L and DOX $(-) ; n=10$. b Representative flow cytometry scatter plots of EPC2T cells with NOTCH3-targeted or non-silencing (NS) control shRNA. NOTCH3 shRNA increased CD44H cells (lower right quadrant) to $29.8 \pm 0.4 \%$ s.d. as compared to $0.4 \pm 0.1 \%$ s.d. in NS control $(P<0.0001$ by Student's $t$-test, $n=3)$. c qRT-PCR analysis for indicated genes comparing EPC2T cells with or without NOTCH3 knockdown. mRNA level for each gene in NS control cells was set to 1 . ${ }^{\star} P<0.05$ vs. NS, $n=3$. d Schematic of NOTCH3 second intron (N3Int2) region and ChIP PCR primers in $\mathbf{f}$ and Supplementary Fig. 4e. Primers amplify the region lacking ZEB1 or CSL-binding sites. e Transfection assays for $p G L 3-N 3$ Int2-luc reporter activity with or without ectopic ZEB1 or ZEB2 expression. bla, empty vector control for ZEBs. ${ }^{\star} P<0.05$ vs. bla and $p G L 3-$ luc (empty reporter); ${ }^{\#} P<0.05$ vs. bla and $p G L 3-N 3$ Int2-luc; ns not significant vs. bla and $p$ GL3-N3Int2-luc; $n=4$. $\mathbf{f}$ ChIP assays for ZEB1 binding to N3Int2 region in purified CD44L and CD44H cells. CD44L cells were stimulated with TGF $\beta$ for 14 days to induce $C D 44 \mathrm{H}$ cells. ${ }^{\star} P<0.0005$ vs. CD44L and IgG and TGF $\beta(-) ;{ }^{\#} P<0.005$ vs. CD44H and IgG and TGF $\beta(-) ;{ }^{\star \star} P<0.05$ vs. CD44L and anti-ZEB1 and TGF $\beta(+) ;$ ns not significant vs. IgG and TGF $\beta(-) ; n$ =3. $\mathbf{g}$ Representative flow cytometry scatter plots of EPC2T cells with ZEB1-targeted or NS control shRNA. ${ }^{\star} P<0.05$ vs. NS and TGF $\beta(-)$; ${ }^{*} P<0.0001$ NS and TGF $\beta(+)$; ns not significant vs. NS and TGF $\beta(-) ; n=3$. In $\mathbf{b}, \mathbf{g}$, flow cytometry was done 7 days following lentivirus infection. All bar diagrams represent mean \pm s.d. At least three independent replicates were performed for all experiments. Fisher's exact test was used for percentage comparisons in a. Student's $t$-test was used for paired data comparisons in $\mathbf{b}, \mathbf{c}, \mathbf{f}$. ANOVA with Tukey's post hoc test was used for multiple comparisons in $\mathbf{e}, \mathbf{g}$ 
a

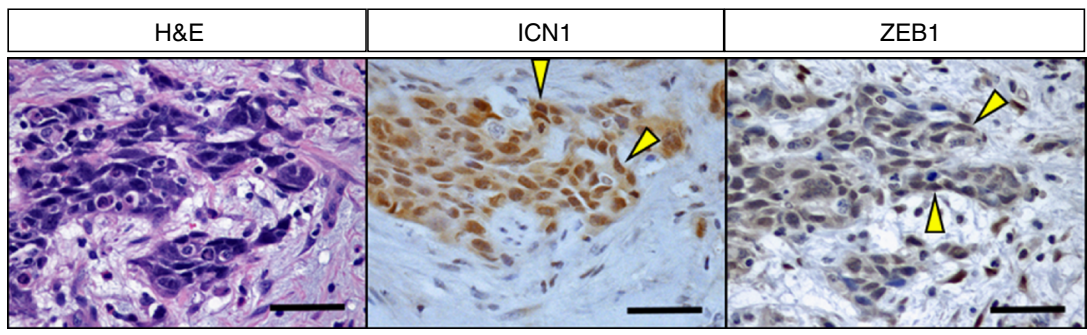

b

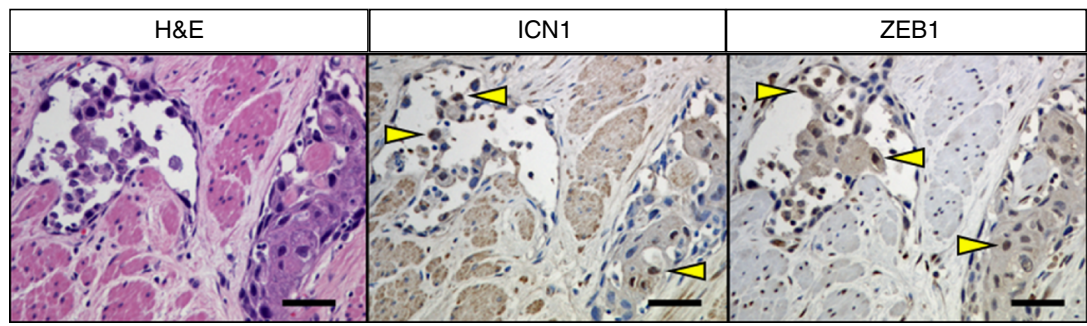

C

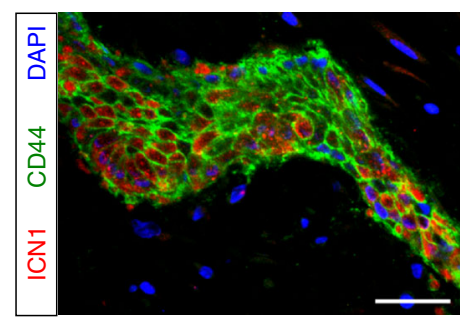

d

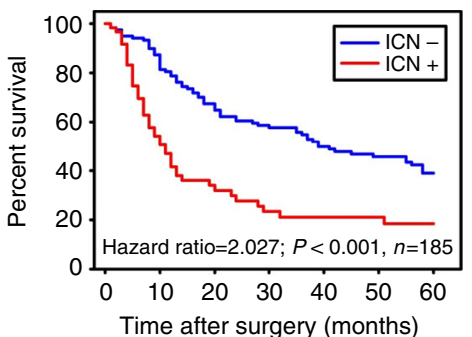

e

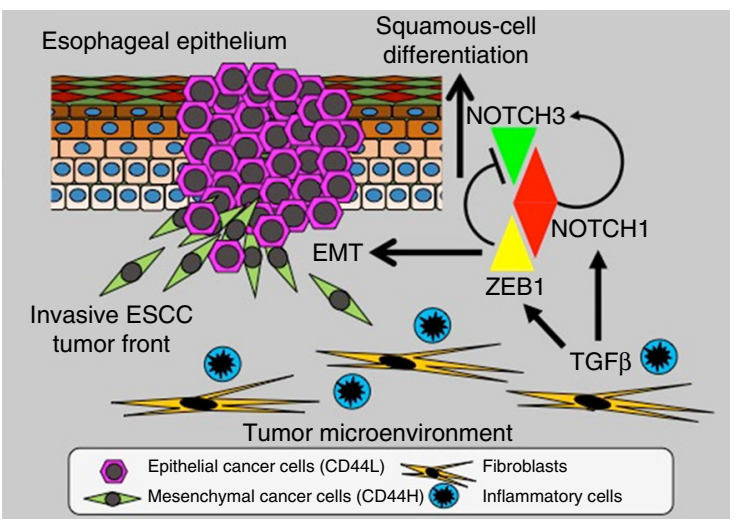

Fig. 7 NOTCH1 activation and ZEB1 expression in invasive ESCC predicts poor prognosis. a, b Representative IHC images for ICN1 and ZEB1 in ESCC cells in a deep invasive tumor nest and ESCC cells invading into lymphatic vessels. a ESCC \#55; b ESCC \#62 in Supplementary Data 1. c Representative IF image for ICN1 and CD44 in invasive ESCC cells. Scale bars, $50 \mu \mathrm{m}$ in a-c. d Survival curve for 185 post-surgical ESCC patients with or without ICN1 expression at the tumor invasive front. e Model of the role of Notch1 in EMT in ESCC. Notch1 activation promotes tumorigenicity and heterogeneity in SCC via EMT. Notch1 drives squamous-cell differentiation by inducing Notch3 in normal squamous epithelia as well as SCC cells. Notch3 limits EMT. In response to TGF $\beta$ from the tumor microenvironment (e.g., cancer-associated fibroblasts and inflammatory cells), however, transcriptional repression of Notch3 by ZEB1 permits Notch1-mediated induction of $\mathrm{CD} 44 \mathrm{H}$ cells via EMT. Notch activation may result in the generation and maintenance of CD44H cells possessing mesenchymal properties and enhanced malignant potential. EMT allows neoplastic cells to cope with stress during carcinogenesis and disease progression (e.g., genotoxic stress induced by 4NQO). CD44H cells produce pro-tumorigenic cytokines (e.g., IL-6) and tissue remodeling factors (e.g., MMP13, LOX, and POSTN) (Supplementary Fig. 3e)

ZEB2 expression upon N3Int2-modulated transcriptional activity. ZEB1 specifically suppressed basal $p G L 3-N 3$ Int2-luc reporter activity and also abrogated reporter activation via DOX-induced ectopic ICN1 (Fig. 6e; Supplementary Fig. 4d). Chromatin immunoprecipitation (ChIP) analysis revealed enrichment of ZEB1 binding to the N3Int2 region in purified CD44L cells with TGF $\beta$ stimulation and purified $\mathrm{CD} 44 \mathrm{H}$ cells without TGF $\beta$ stimulation (Fig. 6f). TGF $\beta$ did not prevent ICN1 from binding to the N3Int2 region (Supplementary Fig. 4e), suggesting that ZEB1 binding may predominate over ICN1 to repress NOTCH3 transcription. $Z E B 1$-directed shRNA attenuated TGF $\beta$-induced $\mathrm{CD} 44 \mathrm{H}$ cell expansion, further implicating ZEB1 as a critical positive regulator in EMT-mediated generation of $\mathrm{CD} 44 \mathrm{H}$ cells (Fig. 6g). 
Notch1 at SCC invasive fronts predicts poor patient prognosis. To demonstrate further the importance of Notch1 activity in pathogenesis of human SCCs, we analyzed surgically procured tissue samples (Supplementary Data 1) by immunohistochemistry (IHC) with two independent NOTCH1 antibodies, both detecting nuclear NOTCH1 at a $95.24 \%$ concordance rate $(\kappa=0.664,95 \%$ CI: $0.008-1.0$, Kappa statistic, $n=21$, Supplementary Fig. 5). ICN1 (ICN1 $1^{\text {Val1744 }}$ ) was readily detectable in normal esophageal squamous epithelia $(n=102)$ as described ${ }^{4}$ and was upregulated in superficial precancerous as well as early invasive ESCC lesions (Supplementary Fig. 6). Within tumors invading into submucosa or muscularis propria, most SCC cells $(>80 \%)$ did not express ICN1 with or without NOTCH1 mutations ${ }^{6,7}$; however, a small subset of SCC cells expressed ICN1 in the invasive tumor front (35.3\% for HNSCC, $n=17 ; 37.0 \%$ for ESCC, $n=227$ ) (Fig. $7 \mathrm{a}$; Supplementary Fig. 6). Accompanied by desmoplastic stroma, ICN1-positive invasive SCC often displayed spindle-shaped cell morphology and ZEB1 co-localization (17.1\%, $n=174)$ (Fig. 7a; Supplementary Fig. 7) with ICN1 and ZEB1 expression being correlated $(\rho=0.31, P<0.0001$, Pearson correlation). When stratified by co-localization status, expression of ICN1 and ZEB1 was relatively uncorrelated for subjects lacking co-localization ( $\rho$ $=-0.1, P=0.2$, Pearson correlation), while expression was correlated more strongly for those showing co-localization $(\rho=0.37$, $P<0.05$, Pearson correlation, $n=30$ ). Additionally, these cells were often found invading into lymphatic vessels (Fig. 7b). NOTCH3 expression was low, if not absent, in SCC cells with concurrent ICN1 and ZEB1 expression (Supplementary Fig. 7). Moreover, ICN1 was expressed in a subset of ESCC cells with elevated CD44 expression (Fig. 7c). Evaluation of ICN1 in relation to clinicopathological data revealed that ICN1 was significantly associated with increased lymph node and distant metastases and advanced disease stages (Supplementary Table 1). Finally, analyses of clinical databases revealed that ICN1 expression at the invasive tumor front predicts independently for poor prognosis (Fig. 7d; Supplementary Table 2).

\section{Discussion}

The current study highlights an oncogenic role for Notch1 in SCC via EMT-mediated induction of $\mathrm{CD} 44 \mathrm{H}$ cells with enhanced malignant potential (Fig. 7e). We demonstrate that Notch1 and Notch3 may have opposing functions to allow expansion of $\mathrm{CD} 44 \mathrm{H}$ cells both in vitro and in vivo. Moreover, we find that TGF $\beta$ guides Notch 1 to drive EMT via a previously undescribed robust shift in the spectrum of Notch1 target genes including Notch3, which is essential in squamous-cell differentiation ${ }^{4}$ and senescence $^{12,}{ }^{48}$. Given the microRNA-mediated crosstalk between Notch signaling and ZEB1 ${ }^{33}, 49$, our findings indicate that direct repression of NOTCH3 by ZEB1 is a novel mechanism through which ZEB1 may influence Notch1-mediated cell fate determination. Activated Notch1 (i.e., ICN1) interacts with TGF $\beta$ downstream effector SMAD3 ${ }^{45}$. ZEB1 also binds physically to SMAD3 to enhance TGF $\beta$-mediated transcription ${ }^{34}$. Additionally, ZEB1 promotes tumorigenicity by repressing stemnessinhibiting microRNAs in pancreas cancer ${ }^{50}$, where ZEB1 induction and EMT have been implicated as the earliest event preceding tumor formation in cell-lineage tracing experiments ${ }^{51}$. Taken together, these preclinical studies suggest that Notch 1 and ZEB1 may cooperate to promote carcinogenesis and SCC progression via TGF $\beta$-mediated EMT and offer the TGF $\beta-Z E B 1-N o t c h 1$ axis as potential therapeutic target in SCC.

Despite a well-established role for the Notch pathway in regulation of varied cell fates in a tissue-dependent and contextdependent manner, the molecular mechanisms governing Notchmediated cell fate determination have yet to be fully elucidated. In non-transformed esophageal epithelium, Notch1-dependent transcriptional upregulation of Notch 3 mediates squamous-cell differentiation as shown by us ${ }^{4}$. In the current study, we find Notch1 facilitates EMT-mediated expansion of CD44H cells with enhanced malignant potential. This raises the question of how Notch1 may act as a signal to induce both differentiation and dedifferentiation (i.e., EMT) in squamous epithelium. One potential explanation for this dichotomy is the influence of tissue microenvironment upon Notch-mediated cell fate determination. Indeed, microarray gene expression analysis in ICN1overexpressing transformed esophageal keratinocytes indicates that TGF $\beta$ induces a dramatic shift in the spectrum of Notch1 target genes from a profile enriched for genes involved in squamous-cell differentiation to one consistent with EMT. Notch1 may also facilitate senescence in response to TGF $\beta$ in esophageal keratinocytes with intact cell cycle checkpoint functions ${ }^{12}$. The complexity in the role of Notch signaling in tumor biology is echoed by that of TGF $\beta$ as intratumoral cancer cell heterogeneity represents cancer cells that can respond to TGF $\beta$ and those cannot, the latter emerging during disease progres$\operatorname{sion}^{52}$. Thus, like TGF $\beta$, Notch1 may have differential roles in cancer initiation and development. Besides TGF $\beta$, hypoxia and inflammation may activate Notch1 to promote EMT in the tumor microenvironment via transcriptional factors such as HIF $1 \alpha^{17}$ and $\mathrm{NF}-\kappa^{53}$, respectively. Potential influence of these factors upon Notch1-mediated cell fates warrants further investigation in SCC as well as squamous epithelia under eosinophilic esophagitis where EMT and Notch3 downregulation are implicated (P.M.C. and H.N., unpublished observation).

While our own published findings and those of others have implicated Notch1 as a tumor promoter in SCCs ${ }^{12,54-56}$, the current study is the first to demonstrate in vivo that Notch1 facilitates tumor initiation by converting CD44L cells to highly tumorigenic $\mathrm{CD} 44 \mathrm{H}$ cells with mesenchymal traits, complementing the earlier studies implicating EMT and ZEB1 in the CD44L-to-CD44H transition $24,28,57$. While Notch1 activation and EMT were detected at the invasive tumor front, SCC cells with epithelial characteristics were commonly present in xenograft tumors, 4NQO-induced tumors, and human SCC samples. In our cell-lineage tracing experiments utilizing $\mathrm{K} 5 \mathrm{Cr} \mathrm{e}^{\mathrm{ERT}}$; R26tdTomato ${ }^{l s l} / / s l$ mice carrying $4 \mathrm{NQO}$-induced tumors, complete loss of epithelial characteristics was uncommon, albeit present (Supplementary Fig. 1d). Despite enhanced expression of mesenchymal-associated genes in EpCAM $^{\text {neg }}$ cells (Fig. 1c, d), most tumors displayed a broad range of continuous EpCAM expression (Supplementary Fig. 1c), suggesting a highly heterogeneous and plastic nature of SCC cells. Besides squamous-cell differentiation, Notch3 may be permissive for mesenchymal-epithelial transition or epithelial-mesenchymal hybrid characteristics of cancer cells by limiting EMT ${ }^{58,59}$. These findings have potential clinical impact as reducing $\mathrm{CD} 44 \mathrm{H}$ cells may delay disease progression or post-therapeutic recurrence and increase sensitivity to chemotherapeutic agents, providing a platform for new human clinical trials. Given the interplay between Notch1 and Notch3 in regulating esophageal cell fate, the use of pan-Notch inhibitors may prove problematic. Indeed, genetic Notch inhibition via DNMAML1 expression accelerates carcinogen-mediated esophageal tumor initiation, growth and invasion in vivo ${ }^{60,61}$. Tumor initiation by $\mathrm{CD} 44 \mathrm{H}$ cells may be independent of Notch because purified $\mathrm{CD} 44 \mathrm{H}$ cells gave rise to tumors with or without DOX-induced ICN1 expression or in the presence of DNMAML1 (Fig. 3c). Interestingly, Notch inhibition by DNMAML1 permits clonal immortalization and expansion of murine esophageal epithelial cells ${ }^{61}$. Thus, Notch1 activation is essential for the induction of $\mathrm{CD} 44 \mathrm{H}$ cells while it may be dispensable for their maintenance and tumor initiation by $\mathrm{CD} 44 \mathrm{H}$ 
cells. Such a premise is corroborated by ICN1 expression localized to tumor invasive fronts, but not in the majority of expanding ESCC cell populations (Supplementary Fig. 6). Notch signaling can be selectively modulated by Notch receptor paralog-specific antibodies $^{62,63}$. TGF $\beta$ signaling can be also regulated by TGF $\beta$ inhibitors ${ }^{64}$. Notably, the observed inhibitory effects of anti-TGF $\beta$ antibody upon tumor progression in mice (Fig. 4) may involve TGF $\beta$ downstream effectors other than Notch1, representing a limitation of the current study. Since TGF $\beta$-induced EMT involves autophagy in ESCC cells where pharmacological inhibition of autophagy flux by chloroquine decreases $\mathrm{CD} 44 \mathrm{H}$ cells, an alternative approach to target EMT and $\mathrm{CD} 44 \mathrm{H}$ cells $^{30}$. Future studies evaluating the efficacy of these agents in SCC therapy are currently underway in preclinical models.

Technical innovation in this study includes use of a HNSCC PDX model as well as a cell-lineage tractable mouse model of ESCC through which EMT was for the first time documented unequivocally in SCC cells in situ. Additionally, we have developed an esophageal 3D organoid platform that recapitulates esophageal tissue architecture ex vivo and can be utilized to model esophagitis ${ }^{65}$. Here, we apply this model system to SCC, generating murine ESCC-derived 3D organoids for the first time (Fig. 1e). Upon ex vivo culture, single-cell-derived 3D organoids from 4NQO-induced dysplasia and ESCC tumors display morphological characteristics of neoplastic epithelium, including nuclear atypia, perturbed squamous-cell differentiation and evidence of EMT, which are maintained upon organoid passaging (K.A.W., P.M.C., and H.N., unpublished observation). We have also successfully performed ex vivo Cre-mediated recombination in 3D organoids generated from neoplastic esophageal keratinocytes of 4NQO-treated Notch $1^{\text {loxP/loxP }}$ mice, demonstrating that Notch1 is required for organoid formation and EMT-like features in neoplastic 3D organoids (Figs. 2f, 3e). Notch1 deletion in the murine skin promotes SCC development in a non-cell autonomous fashion due to inflammation associated with epidermal barrier defects ${ }^{5}$, limiting the assessment of the cell-autonomous oncogenic role of Notch1 in vivo. The 3D organoid system provides insights about cell-autonomous oncogenic functions of Notch1 in the absence of inflammatory milieu ex vivo, complementing in vivo experiments. The $4 \mathrm{NQO}$ model requires a long-term 4NQO administration (16 weeks) where tumors arise 6-9 weeks after $4 \mathrm{NQO}$ withdrawal during the observation period; however, it is not precisely known when malignant transformation occurs in this model. The single-cell-derived 3D organoids have a potential to detect neoplastic changes in a more sensitive and quantitative manner than conventional morphological tissue assessment. Such a study is underway. Once an appropriate window of $4 \mathrm{NQO}$-induced malignant transformation is determined, our cell-lineage traceable mice can be utilized for genetic ablation of Notch1 following 4NQO administration to elucidate how Notch1 may exert its oncogenic role by promoting EMT and tumor initiation and/or progression in vivo. Alternatively, such mice may be treated with Notch1-specific antagonistic antibody ${ }^{63}$ or anti-Notch3 agonistic antibody ${ }^{62}$, the latter can be used for targeted activation of Notch3. While these findings identify 3D neoplastic organoids as sustainable resource for functional and mechanistic investigations into the biology of ESCC, it remains to be determined how faithfully organoid genetics and biology mimic that of the tissue from which they are derived upon extended ex vivo culture. Ongoing studies include procuring of a bank of human patient-derived ESCC 3D organoids that may be utilized as an experimental platform for discovery and validation of novel translational applications for prognosis and therapy in the setting of personalized medicine.

Taken together, the current study supports a tumor-promoting role of Notch1 in SCC while also providing novel mechanistic insight into how the local tissue microenvironment may influence Notch-mediated cell fate determination.

\section{Methods}

Patients and tissue samples. Surgically removed tissue samples (Supplementary Data 1) were described previously ${ }^{11,66-68}$ or newly procured at Kagoshima University Hospital in accordance with Institutional Review Board standards and guidelines. Informed consent was obtained from all human subjects. Most samples were available as tissue microarrays (TMAs) ${ }^{67,68}$ containing primary ESCC $(n=$ $171)$, carcinoma in situ (CIS; $n=9)$, IEN $(n=7)$, and normal mucosa $(n=114)$. Samples with NOTCH1 mutations $\left(n=4 ; \mathrm{E} 755^{*}, \mathrm{D} 469 \mathrm{Y}, \mathrm{R} 1279 \mathrm{D}\right.$, and D1457G) and wild-type NOTCH1 $(n=15)$ were identified by DNA sequencing (SRP072948) ${ }^{69}$. Given the limitation of TMAs to assess intratumoral cancer cell heterogeneity ${ }^{70}$, we examined whole paraffin blocks $(n=244)$ by Hematoxylin and Eosin $(\mathrm{H} \& \mathrm{E})$ staining and IHC, as described below, following preliminary analysis with TMAs. Survival analysis was done on IHC data for 185 ESCC patients who did not receive chemotherapy or radiation therapy prior to surgery.

\section{Esophageal epithelial cell-lineage traceable mice and 4NQO treatment. The} K5Cre $e^{E R T 2}$ transgenic mouse strain ${ }^{68}$ was intercrossed with 226 tdTomato $^{l s l} /$ sl $l$ (Jackson Laboratory, Bar Harbor, ME) carrying the homozygous Rosa26 locus with knocked-in tdTomato fluorescent protein as a reporter under the loxP-stop-loxP sequence. The resulting $\mathrm{K} 5 \mathrm{Cr} e^{E R T 2}$;R26tdTomato ${ }^{\text {Isl/wt }}$ mice were crossed with

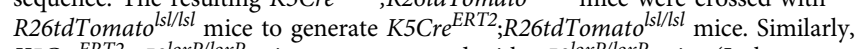
K5Cre ${ }^{E R T 2} ; p 53^{\text {loxP/loxP }}$ mice were generated with $p 53^{\text {loxP/loxP }}$ mice (Jackson Laboratory). R26tdTomatolsl/sl mice were also crossed with $p 53^{\text {loxP/loxP }}$ to generate R26tdTomato ${ }_{\text {lsl/wt }}$; $53^{\text {loxP } / w t}$ mice, which were then crossed to generate R26tdTomato $^{\text {lsl/lsl }} ; p 53^{\text {loxP/loxP }}$ mice. The K5Cre $e^{E R T 2} ; p 53^{\text {loxP/loxP }}$ mice were further crossed

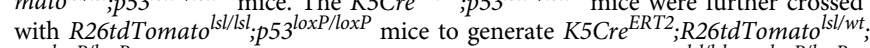
$p 53^{\text {loxP/loxP }}$. These mice were further crossed with R26tdTomato lsl/lsl $_{p}$. $53^{\text {loxP/loxP }}$ to

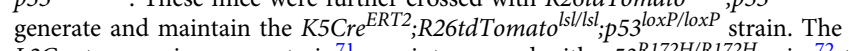
L2Cre transgenic mouse strain ${ }^{71}$ was intercrossed with $p 53^{R 172 H / R 172 H}$ mice $^{72}$ to generate L2Cre; $553^{R 172 H / w t}$ mice.

We administered TAM (Sigma-Aldrich, St. Louis, MO; $0.25 \mathrm{mg} / \mathrm{g}$ body weight) via oral gavage to $3-4$-month-old $\mathrm{K}^{\mathrm{CCr}} \mathrm{ERT2}^{E R 26 t d T o m a t o}{ }^{l s l / l s l}$ and $\mathrm{K} 5 \mathrm{Cre} \mathrm{ERT2}^{E R}$; R26tdTomato ${ }^{\text {sll/lsl }}$; $p 53^{\text {loxP/loxP }}$ littermates 2 weeks before starting 4-Nitroquinoline $\mathrm{N}$-oxide (4NQO) (Sigma-Aldrich) treatment. Mice received $100 \mu \mathrm{g} / \mathrm{ml} 4 \mathrm{NQO}$ in $2 \%$ propylene glycol (MP Biomedicals, Solon, $\mathrm{OH}$ ) in drinking water for 16 weeks and were followed up for 8-10 weeks (or earlier if mice were ill) after 4NQO withdrawal as described ${ }^{73}$. To inhibit TGF $\beta$ receptor-mediated signaling, mice were treated for 2 weeks with either anti-TGF $\beta$ antibody 1D11 (intra-peritoneally), neutralizing all three isoforms of TGF $\beta^{46}$ (a gift of Dr. Singhal, University of Pennsylvania; $3 \mathrm{mg} / \mathrm{kg}$, three times per week) or control IgG (intra-peritoneally) with injections initiated 4 weeks following $4 \mathrm{NQO}$ withdrawal. At the time of sacrifice, one half of the dissected esophagus or a tumor (if visible macroscopically) from each mouse was fixed in $4 \%$ paraformaldehyde and paraffin-embedded for morphological analyses. The other half of esophagi and tumors was dissociated as described previously ${ }^{74}$ and cell suspensions were subjected to flow cytometry or FACS and organoid formation assays. All experiments were done under approved protocols from the University of Pennsylvania Institutional Animal Care and Use Committee (IACUC). Sample size for groups were projected based on data from pilot studies. Animals were only excluded from analyses in event of death from procedure-related causes (e.g., death following oral gavage) that were unrelated to experimental differences between groups. Animals were randomized to treatment groups with consideration given to representation of both sexes. Investigators were informed of groups during treatment phase of experiments. Upon processing, tissues were given a unique identifier to blind investigators during analyses and outcome assessments.

Xenograft transplantation experiments. Xenograft transplantation experiments were done as described ${ }^{11}, 12$. In brief, $1-5 \times 10^{6}$ cells were suspended in $50 \%$ Matrigel and implanted subcutaneously into the dorsal skin of female athymic nu/ $n u$ mice (4-6 weeks old; Charles River Breeding Laboratories). PDX tumor transplantation was performed as previously described with fragments of passaged PDX tumors implanted under the dorsal skin of NOD/SCID/IL2 receptor $\gamma$-chaindeficient (NSG) mice ${ }^{75}$. Tumor growth was monitored and mice were sacrificed 6-10 weeks after inoculation for flow cytometry and histopathological analyses. One half of each tumor was fixed in formalin and embedded in paraffin blocks. The other half of each tumor was enzymatically dissociated and subjected to flow cytometry and FACS for serial transplantation experiments where $10-10^{3}$ cells were injected into recipient mice to determine tumor formation rates. For tetracycline/DOX-inducible (TetOn) transgene expression in xenograft tumors, mice were fed daily with DOX-containing pellets $(20 \mathrm{mg} / \mathrm{kg})$. To inhibit TGF $\beta$ receptormediated signaling, mice were treated for 2 weeks with either anti-TGF $\beta$ antibody $1 D 11$ (intra-peritoneally) $(3 \mathrm{mg} / \mathrm{kg}, 2-3$ times per week) or control IgG (intraperitoneally), beginning when tumor volume reached $60 \mathrm{~mm}^{3}$. All experiments were approved by the University of Pennsylvania IACUC or under Wistar Institute IACUC protocols 112652/112655. Sample size for groups were projected based on previous xenograft studies then adjusted following acquisition of data in initial 
experiment. Animals were only excluded from analyses in event of death from procedure-related causes (e.g., sepsis) that were unrelated to experimental differences between groups. Animals were randomized by cage upon arrival. Investigators were informed of groups during treatment phase of experiments. Upon processing, tumors were given a unique identifier to blind investigators during analyses and outcome assessments.

DNA constructs. A lentiviral vector pTRIPZ expressing DOX-inducible shRNA directed against human NOTCH1 (Notch1-A; V2LHS_149557 and Notch1-B; V3LHS_637133) and pTRIPZ-NS carrying a non-silencing scrambled sequence (RHS4346) (GE Dharmacon, Lafayette, CO) were purchased. Other shRNA sequences in pGIPZ (GE Dharmacon) targeting NOTCH3 (Notch3-A; V2LHS_229748 and Notch3-B; V2LHS_93017), ZEB1 (ZEB1-A; V2LHS_116663 and ZEB1-B; V2LHS_116659), and a non-silencing scrambled sequence (RHS4348) were used as described previously $11,12,19$.

To generate a lentiviral vector pTR-tRFP expressing constitutively turbo RFP $(t R F P)$ under ubiquitin $\mathrm{C}(\mathrm{UBC})$ promoter, a DOX-inducible gene expression vector pTRIPZ-MCS ${ }^{12}$ was modified by removal of BamHI and LoxP sites and a subsequent insertion of new multiple cloning sites (MCS) comprising 5'-AgeIBamHI-SnaBI-EcoRI-XhoI-MluI-3', resulting in the creation of pTRIPZ-MCS2. We then amplified an open reading frame (ORF) for turbo RFP ( $t R F P$ ) by PCR using pTRIPZ-NS (RHS4743, GE Dharmacon) as a template with primers $5^{\prime}$ AGCGCTAGCGCCACCATGAGCGAGCTGATC- ${ }^{\prime}$ ' and $5^{\prime}$-AGCGCGGCCGC TTATCTGTGCCCCAGTTTGCTAGG-3'. Following removal of rtTA-encoding ORF flanked by NheI and NotI sites, the purified $t R F P$ ORF was subcloned into the NheI and NotI sites of pTRIPZ-MCS2, resulting in creation of pTR-tRFP. To generate a lentiviral vector carrying $8 \times C S L-G F P$ reporter, an ORF for GFP was isolated from pBABE-zeo-GFP ${ }^{19}$ and ligated into the EcoRI and XhoI sites of pTRtRFP, generating pTR-tRFP-GFP. A DNA fragment containing a concatemer of eight copies of CSL-binding sites fused to minimal SV40 (mSV40) promoter was isolated from $8 \times C S L-l u c^{76}$ by PCR with primers $5^{\prime}$-AGCTCTAGACTCTTACGC GT GCTAGCTCGA-3' and 5'-AGCACCGGTTTTGCAAAAGCCTAG GCCTCC A- $3^{\prime}$ to replace the sequence containing tetracycline response elements and minimal CMV promoter from pTR-RFP-GFP at the $\mathrm{XbaI}$ and AgeI sites, resulting in a creation of pTR-RFP- $8 \times$ CSL-GFP.

To delete human NOTCH1 via Crispr/Cas9-mediated gene editing, five candidate guide RNA (gRNA) sequences were subcloned into lentiCRISPR v1 (gift of a gift from Feng Zhang; Addgene, plasmid \#52961) at its BsmBI sites and used as described $^{77}$. gRNAs 1,2 , and 5 targeting chromosome 9 NOTCH1 exon 14 (EGFlike region at $139,407,894-139,407,913$ ) or exon 19 (EGF-like region at $139,403,465-139,403,484$ ) and exon 34 (PEST domain at

$139,390,539-139,390,558)$, respectively, were found to be equally effective to delete NOTCH1.

To generate a retroviral vector BABE-zeo-FLAG-DNMAML1, we amplified an Flag-MAML1 ${ }^{1-302}$ ORF by PCR using pFLAG-CMV2-MAML1 (1-302) (a gift from Dr. Wu, University of Florida) as a template with primers $5^{\prime}$-GCACGGAT CCGCCACCATGGACTACAAAGACGATGA CGACAAG- ${ }^{\prime}$ and $5^{\prime}$-GCACGTC GACCTAGAATTCCGTCTTAATATTAATGTCCTGTGCC- $3^{\prime}$ and subcloned at the BamHI and SalI sites of pBABE-zeo ${ }^{78}$. To generate pBABE-bla-ZEB1 and pBABE-bla-ZEB2, ZEB1 and ZEB2 ORFs in pCR-BluntII-TOPO were purchased from GE Dharmacon (clone 40036600 for ZEB1 and clone 40124124 for ZEB2) The ZEB1 and ZEB2 ORFs were amplified by PCR with primers 5'-CGCTGGA TCCGCCGCCACCATGGCGGATGG CCCCAGGTG-3' and $5^{\prime}$-CGGAGTCGAC TTAGGCTTCATTTGTCTTTTC- $3^{\prime}$ for ZEB1 and $5^{\prime}$-CG CTGGATCCGCCGC CACCATGAAGCAGCCGATCATGGC- ${ }^{\prime}$ ' and $5^{\prime}$-CGGAGTCGACTTACATGC CATCTTCCATATTG-3' for ZEB2, respectively, and subcloned at the BamHI and SalI sites of pBABE-bla ${ }^{79}$.

The 2nd intron of NOTCH3 (N3Int2) containing ZEB1-binding sites (488-bp) was amplified by PCR using the input DNA sample from ChIP assay as a template with primers 5'-GGGAGATCTGGTTCCCACGCTCTGCGTCC-3' and 5'-GGGC TCGAGCCGCGCCTGGAATACTGCCG-3'. Then the amplified DNA was subcloned at the XhoI and BglII sites of pGL3 promoter (Promega, Madison, WI), creating a plasmid pGL3-N3Int2-luc. All constructs were verified by DNA sequencing.

Cell culture, genetic modifications, and pharmacological treatments of cells $\mathrm{TE} 11, \mathrm{EN} 60, \mathrm{EPC} 2 \mathrm{~T}$, and derivatives were grown at $37^{\circ} \mathrm{C}$ in a $5 \% \mathrm{CO}_{2}$ atmosphere as described previously ${ }^{12}$. Additional genetic modifications were done via stable gene transfer of the above retroviral and lentiviral constructs as described previously $^{12}$. Stable cell lines were established by drug selection for 7 days with $1 \mu \mathrm{g} / \mathrm{ml}$ of Puromycin (Invitrogen) for genes transduced via pTRIPZ or lentiCRISPR; and $0.5 \mu \mathrm{g} / \mathrm{ml}$ zeocin (Invitrogen) for Flag-MAML1 ${ }^{1-302}$ transduced via pBABE-zeo. Cells transduced with GFP (pGIPZ) or tRFP (pTR-tRFP) were selected for the brightest level of fluorescence (top 20\%) by FACS. EPC2T, EN60, and derivatives were kept undifferentiated in Keratinocyte serum-free medium (Invitrogen, Carlsbad, CA) containing a low concentration $(0.09 \mathrm{mM})$ of $\mathrm{CaCl}_{2}$. Squamous-cell differentiation was induced by $0.6 \mathrm{mM} \mathrm{CaCl}_{2}$ (Sigma-Aldrich) as described ${ }^{4}$. Compound E (Calbiochem, La Jolla, CA), a GSI, and SB431542 (Calbiochem), a TGF $\beta$ type I receptor kinase inhibitor were reconstituted in dimethyl sulfoxide (DMSO) (Sigma-Aldrich). Cells were treated with $1 \mu \mathrm{M}$ Compound E or $5 \mathrm{ng} / \mathrm{ml}$ recombinant human TGF $\beta 1$ (R\&D Systems, Minneapolis, MN) as optimized previously ${ }^{4,19,80}$. SB431542 was used at $10 \mu \mathrm{M}$. Dox was used at $1 \mu \mathrm{g} / \mathrm{ml}$ for DOX inducible transgene expression ${ }^{12}$. Phase contrast images were acquired using a Nikon Eclipse E600 microscope. EPC2-hTERT cells and derivatives were estab-
lished and extensively characterized by us ${ }^{12}, 30,74$. TE11 $12,30,81$ and EN60 cells were generous gifts from Dr. Tetsuro Nishihira (Tohuko University, Sendai, Japan) and Dr Hiroshi Shirasawa (Chiba University, Chiba, Japan), respectively, and were extensively characterized by us $12,30,74,83$. The earliest frozen stocks of all cell lines have been stored at the Cell Culture Core of the University of Pennsylvania. We have propagated cells from frozen stocks of original vials that were authenticated by short tandem repeat profiling (ATCC) for highly polymorphic microsatellites to validate the identity of cells by comparing cells at the earliest stocks and those grown $>8-12$ passages. All cell lines undergo routine mycoplasma testing.

Organotypic 3D culture. OTC was carried out as described previously ${ }^{74}$. In brief $0.5 \times 10^{6}$ of epithelial cells were seeded on top of the collagen/Matrigel matrices containing FEF3 human fetal esophageal fibroblasts, and grown in submerged conditions for 4 days. Cultures were then raised to the air-liquid interface for additional 4 days and harvested for morphological assessment. Each OTC experiment was performed in triplicate.

Ex vivo esophageal organoid 3D culture. Esophageal keratinocytes were isolated from vehicle-treated or 4NQO-treated K5Cre ${ }^{E R T 2} ; R 26 t_{d T o m a t o}{ }^{I l / l s l}$ or Notch $1^{\text {loxP/loxP }}$ (Jacskon Laboratories) mice under an IACUC-approved protocol as described previously ${ }^{65,84,85}$. Using 24 -well plates, 5000 cells were seeded per well in $50 \mu \mathrm{l}$ Matrigel. After solidification, $500 \mu \mathrm{l}$ of DMEM/F12 supplemented with $1 \times$ Glutamax, $1 \times$ HEPES, $1 \times \mathrm{N} 2$ Supplement, $1 \times$ B27 Supplement, $0.1 \mathrm{mM} \mathrm{N}$-acetyl-Lcysteine (Sigma-Aldrich), $50 \mathrm{ng} / \mathrm{ml}$ mouse recombinant EGF (R\&D Systems), $2.0 \%$ Noggin/R-Spondin-conditioned media and $10 \mu \mathrm{M}$ Y27632 (Tocris Biosciences, Bristol, UK) were added and replenished every other day. For ex vivo recombination, organoids were cultured in the presence of Adenovirus vector containing Cre recombinase and GFP (University of Iowa Gene Transfer Vector Core). Adenovirus vector containing GFP alone was used as a control. Adenovirus vectors were used at 1:500 at the time of organoid plating. Organoid formation rate was calculated as the percentage of the number of organoids formed at day 7 per total number of cells seeded at day 0 . After 14 days organoids were recovered by digesting Matrigel with Dispase I (BD Biosciences, San Jose, CA; $1 \mathrm{U} / \mathrm{ml}$ ) and fixed overnight in $4.0 \%$ paraformaldehyde. Specimens were embedded in $2.0 \%$ BactoAgar: $2.5 \%$ gelatin prior to paraffin embedding.

RNA isolation, cDNA synthesis, $\mathbf{q R T}-\mathbf{P C R}$, and microarray analyses. RNA isolation, cDNA synthesis, and qRT-PCR were done using StepOnePlus ${ }^{\mathrm{TN}}$ RealTime PCR System (Applied Biosystems) by TaqMan ${ }^{\circledR}$ Gene Expression Assays (Applied Biosystems) for NOTCH1 (Hs01062014 m1), Notch1

(Mm00435249_m1), NOTCH3 (Hs00166432_m1), Notch3 (Mm00435270_m1), IVL (Hs00846307_s1), CK13 (s00999762_m1), CDH1 (Hs00170423_m1), CDH2 (Hs00983062_m1), ZEB1 (Hs00232783_m1), SNAI1 (Hs00195591_m1), and PAI1 (Hs01126606_m1), and SYBR ${ }^{\circledR}$ Green PCR for human ACTB ( $\beta$-Actin) as well as murine $C d h 1$ (5'-TCAAGCTCGCGGATAACCAGAACA- $3^{\prime}$ and $5^{\prime}$ -

ATTCCCGCCTTCATGCAGTTGTTG-3'), Cdh2 (5'-ATGGCCTTTCAAACACA GCCACAG-3' and 5'-ACAATGACGTCCACCCTGTTCTCA-3'), Zeb1 (5'-TGAG CACACAGGTAAGAGGCC- $3^{\prime}$ and $5^{\prime}$-GGCTTTTCCCCAGAGTGCA-3'), Zeb2 (5' ${ }^{\prime}$-TGATAGCCTTGCAAACCCTCTGGA-3' ${ }^{\prime}$ and $5^{\prime}$-TCCTTCATTTCTTCTGG ACCGGCT-3'), Twist (5'-AGCTGAGCAAGATTCAGACCCTCA-3' and 5'-TGC AGCTTGCCATCTTGGAGT-3'), and Gapdh (5'-GGTGGTCTCCTCTGACTTC AACA-3' and 5'-GTTGCTGTAGCCAAATTCGTTGT-3') and as described ${ }^{4}, 11,19$ All PCR reactions were performed in triplicate. The relative level of each mRNA was normalized to ACTB ( $\beta$-actin) for human genes and Gapdh for murine genes as internal controls. Gene array experiments were done using an Affymetrix gene chip (U133+v2.0) (Affymetrix, Santa Clara, CA) as described ${ }^{11,31}$. Data were deposited at the NCBI Gene Expression Omnibus (http://www.ncbi.nlm.nih.gov/ geo) (accession \#GSE37994).

Immunoblot analysis. Whole cell lysates were prepared as described ${ }^{4,11} .20 \mu \mathrm{g}$ of denatured protein was fractionated on a NuPAGE Bis-Tris 4-12\% gel (Invitrogen). Following electrotransfer, Immobilon-P membranes (Millipore) were incubated with primary antibodies for rat monoclonal anti-NOTCH1 5B5 (1:1000; 3447, Cell Signaling, Danvers, MA), rabbit monoclonal anti-ICN1 Val1744 D3B8 (1:1000; 4147, Cell Signaling), rat monoclonal anti-NOTCH3 8G5 (1:1000; 3446, Cell Signaling) mouse monoclonal anti-E-cadherin (1:10,000; 610182, BD Biosciences), mouse monoclonal anti-N-cadherin clone 32 (1:1000; 610920, BD Biosciences), mouse monoclonal anti-Involucrin clone SY5 (1:1000, I9018, Sigma-Aldrich) and mouse monoclonal anti- $\beta$-actin (1:10,000; AC-74, Sigma-Aldrich), and then with the appropriate HRP-conjugated secondary antibody (GE Healthcare, Piscataway, NJ). $\beta$-actin served as a loading control. Uncropped images of blots from primary figures are shown in Supplementary Fig. 8. 
Flow cytometry and FACS. Flow cytometry and FACS were performed as described previously ${ }^{86}$. FACSCalibur or LSRII (BD Biosciences, San Jose, CA) and FlowJo (Tree Star, Ashland, OR) were used for flow cytometry. FACS Vantage SE and FACS Aria II (BD Biosciences) were used to sort purified CD $44^{\text {high }}$-CD2 $4^{\text {low/- }}$ cells $(\mathrm{CD} 44 \mathrm{H})$ and CD44 low/--CD24 $4^{\text {high }}$ cells $(\mathrm{CD} 44 \mathrm{~L})$ from EPC2T, TE11 and EN60 cells, and/or xenograft tumors. Cells were suspended in Hank's balanced salt solution (Invitrogen) containing 1\% BSA (Sigma-Aldrich) and stained with the following antibodies on ice for $30 \mathrm{~min}$ : human PE/Cy7-anti-CD24 clone [ML5] (1:40; 311,120, BioLegend, San Diego, CA), human APC-anti-CD44 clone G44-26 (1:20; 559,942, BD Biosciences), human FITC-anti-CD326 (EpCAM) clone HEA125 (1:50; 130-089-113, Miltenyi Biotec, San Diego, CA) and/or mouse APC-Cy7anti-CD326 (EpCAM) Clone G8.8 (1:50; 118,217, BioLegend). 4',6-diamidino-2phenylindole (DAPI; $2 \mu \mathrm{g} / \mu \mathrm{l}$; Invitrogen) was used to assess viability. CD44T cells were defined as CD $44^{\text {high }}$-CD24 $4^{\text {High }}$. To purify CD44L and CD $44 \mathrm{H}$ cells from TE11 and EN60 xenograft tumors, tumors were minced into $1 \mathrm{~mm}^{3}$ pieces and incubated in Dulbecco's Modified Eagle Medium (DMEM; Invitrogen) containing $1 \mathrm{mg} / \mathrm{ml}$ collagenase I (Sigma-Aldrich) at $37^{\circ} \mathrm{C}$ for $90 \mathrm{~min}$. Following centrifugation, residual tissue pieces were digested in $0.05 \%$ trypsin-EDTA (Invitrogen) at 37 ${ }^{\circ} \mathrm{C}$ for $10 \mathrm{~min}$ and then with $1 \mathrm{U} / \mathrm{ml}$ Dispase (BD Biosciences) and $100 \mu \mathrm{g} / \mathrm{ml}$ DNase I (\#10104159001, Roche) at $37^{\circ} \mathrm{C}$ for $10 \mathrm{~min}$. Dissociated tumor cells were filtered with a $40 \mu \mathrm{m}$ cell strainer (BD Biosciences) and washed prior to incubation with antibodies. Cancer cells were distinguished from host-derived stromal cells by tRFP expression detected concurrently. In $8 \times$ CSL-GFP reporter assays, tRFPpositivity was used to identify human cells while GFP expression was monitored to evaluate Notch activity. In tissue from $4 \mathrm{NQO}$-treated mice and controls, tdTomato expression was used to isolate epithelial cells that had undergone $\mathrm{K} 5 \mathrm{Cre} \mathrm{ERT2}^{\mathrm{E}}$ mediated recombination following TAM treatment. Following dissection, esophagi were incubated with $1 \mathrm{U} / \mathrm{ml}$ Dispase for $5 \mathrm{~min}$ at $37^{\circ} \mathrm{C}$ then epithelial layer was peeled from underlying stroma using fine tipped forceps. Epithelia were then incubated in $0.25 \%$ trypsin two times for $5 \mathrm{~min}$ at $37^{\circ} \mathrm{C}$ with agitation to liberate keratinocytes. Trypsin was quenched with soybean trypsin inhibitor then liberated cells were filtered with a $40 \mu \mathrm{m}$ cell strainer prior to staining. Flow cytometry was repeated for each genotype and condition at least three times.

Immunofluorescence (IF) and IHC. IF and IHC for NOTCH1, NOTCH3, ZEB1, E-cadherin, CD44, and tdTomato were done as described previously $4,11,12$. IHC for Notch1 was done with two independent anti-NOTCH1 antibodies, namely, polyclonal rabbit polyclonal anti-NOTCH1 (ab27526; Abcam; 1:250) and rabbit polyclonal anti-ICN1 1 1744 (2421; Cell Signaling; 1:100), both detecting cytoplasmic and nuclear expression of NOTCH1. The following primary antibodies were used for IHC and/or IF: rabbit polyclonal anti-NOTCH3 (ab23426; Abcam; 1:250), rabbit polyclonal anti-ZEB1 (a generous gift from Dr. Darling, University of Louisville; 1:100), mouse monoclonal anti-E-cadherin (610182; BD Biosciences; 1:100), rabbit polyclonal anti-RFP (ab34771, Abcam; 1:100). IHC signals were developed using the diaminobenzidine substrate kit (Vector Laboratories, Burlingame, CA) following incubation with secondary anti-mouse IgG (Vector; 1:600 at $37^{\circ} \mathrm{C}$ for $30 \mathrm{~min}$ ) or anti-rabbit IgG (Vector; $1: 600$ at $37^{\circ} \mathrm{C}$ for $30 \mathrm{~min}$ ), and counterstained with Hematoxylin (Fisher Scientific CS401-1D). For IF, Cyanine Cy2-conjugated or Cyanine Cy3-conjugated affinity-purified anti-mouse or antirabbit IgG (Jackson Immuno-Research; 1:600) was used for signal detection by incubating at $37^{\circ} \mathrm{C}$ for $30 \mathrm{~min}$, and cell nuclei were counterstained by DAPI (Invitrogen; 1:10,000). Stained objects were examined with a Nikon E600 microscope and imaged with a digital camera. The staining was assessed independently by pathologists (S.N. and A.K.S.), and the intensity was expressed as negative (0), marginally positive (0.5), weakly positive (1), moderately positive (2), or strongly positive (3). Both antibodies detect cytoplasmic and nuclear NOTCH1 at high concordance rates, although anti-ICN1 ${ }^{\mathrm{V} 1744}$ antibody was more sensitive than ab27526 to detect nuclear Notch1 expression (Supplementary Fig. 5). The ab27526 antibody recognizes a full-length NOTCH1 expressed on the cell surface in normal squamous epithelia ${ }^{4}$ as well as well-differentiated SCC cells forming keratin pearl ${ }^{11}$. In this study, we have focused on cytoplasmic and nuclear intracellular Notch1 (ICN1) detected by anti-ICN1 ${ }^{\text {V1744. }}$.

Transient transfection and dual-luciferase assays. Transient transfection of reporter plasmids and luciferase assays was performed as described previously 4,80 . $1 \times 10^{5}$ cells were seeded per well in 24 -well plates $24 \mathrm{~h}$ before transfection. Lipofectamine ${ }^{\mathrm{TM}}$ LTX and Plus ${ }^{\mathrm{TM}}$ reagents (Invitrogen) were used for DNA transfection, according to the manufacturer's instructions. $400 \mathrm{ng}$ of pGL3pN3Int 2 was transfected along with or without $400 \mathrm{ng}$ of pBABE-bla-ZEB1, pBABEbla-ZEB2 of pBABE-bla (empty vector control). $5 \mathrm{ng}$ of phRL-SV40-renilla luciferase vector (Promega) was co-transfected to calibrate the variation of transfection efficiencies among wells. Cells were incubated in the presence or absence of $1 \mu \mathrm{g} / \mathrm{ml}$ DOX to induce ICN1 in cells expressing ICN1 $1^{\text {TetOn }}$ for $48 \mathrm{~h}$ before cell lysis. Alternatively, $5 \mathrm{ng} / \mathrm{ml}$ TGF $\beta 1$ was added at $24 \mathrm{~h}$ after transfection and incubated for an additional $48 \mathrm{~h}$ before cell lysis. Luciferase activities were determined using Dual-Luciferase ${ }^{\mathrm{TM}}$ Reporter Assay system (Promega) and ORION Microplate Luminometer (Berthold Detection Systems, USA, Oak Ridge, TN). The mean of firefly luciferase activity was normalized with the co-transfected renilla luciferase activity. Transfection was carried out at least three times, and variation between experiments was not greater than $15 \%$.
ChIP assay. $1 \times 10^{7}$ cells were treated with $1 \%$ formaldehyde for $10 \mathrm{~min}$ at $37^{\circ} \mathrm{C}$ and quenched with $0.125 \mathrm{M}$ glycine for $5 \mathrm{~min}$ at room temperature. Cross-linked chromatin was sheared into $\sim 500$ bp DNA fragments with Branson Sonifier 250 (Branson, Danbury, CT, USA) and subjected to immunoprecipitation with $2 \mu \mathrm{g} / 10^{6}$ cells with antibody for ZEB1 (sc10572, Santa Cruz), NOTCH1 (sc6014-R, Santa Cruz) or either goat IgG (sc2028, Santa Cruz) or rabbit IgG (sc2027, Santa Cruz) as negative controls. DNA was purified by QIAquick PCR purification kit (QIAGEN, Valencia, CA) and analyzed by real-time PCR using StepOnePlus ${ }^{\text {Th }}$ Real-Time PCR System (Applied Biosystems, Carlsbad, CA). The following primers were used for real-time qPCR: 5'-CCCACAGCCCAACTCGGAGG-3' and 5'-CCGCGCCTGG AATACTGCCG-3' for ZEB1-binding sites at the 2nd intron of NOTCH3, 5'-GC TGGGCGCCGAGGATAG-3' and 5'-AGACCTCGTCCCCATCTCCTAGTC-3' for CSL-binding sites ${ }^{4}$ at the 2nd intron of NOTCH3, $5^{\prime}$-TGCCAAAAGAGGAAG CATAAGTA- $3^{\prime}$ and $5^{\prime}$-TCAAAATCCCTGTGTAGCTGAAT- $3^{\prime}$ for an off-target control $^{4}$ for NOTCHotch3. 5'-CCCTTTCTGATCCCAGGTCT-3' and 5'-GACCT GCACGGTTCTGATTC-3' for a ZEB1-binding site on the of $C D H 1$ promoter ${ }^{87}$, $5^{\prime}$-GAAGTGGCTCCAGTGCTCAAA- ${ }^{\prime}$ and $5^{\prime}$-ATGGCAGTGCATGCCTGTA GT-3' for an off target control for $\mathrm{CDH} 1$ promoter $^{87}, 5^{\prime}$-CGTGTCTCCTCCTC CCATT- $3^{\prime}$ and $5^{\prime}$-CCGCTGTTATCAGCACCAG-3' for a CSL-binding site at the HES 1 promoter ${ }^{88}, 5^{\prime}$-TGGATCCAATCCTATTGCCC-3' and 5'-CGCAGCAGT TGGAAGTGTTT- $3^{\prime}$ for an off target control for $\mathrm{HESI}^{88}$. Data represent three independent experiments.

Statistical analyses. Data from experiments were presented as mean \pm standard error $(n=3-6)$ in real-time RT-PCR, luciferase assays, flow cytometry and IHC labeling index or mean \pm standard deviation $(n=8-12)$ in xenograft transplantation tumor analyses. GraphPad Prism 7 (GraphPad Software, La Jolla, CA) or Stata Version 14 (StataCorp, College Station, TX) software were used for statistical analyses. The two-tailed Student's $t$-test was used for paired comparisons. Fisher's exact test was used for percentage comparisons in xenograft experiments. ANOVA with Tukey's post hoc test was used for multiple pairwise comparisons. $P<0.05$ was considered significant. The Kappa Statistic was used to evaluate concordance between NOTCH1-positive IHC staining as classified by independent antibodies. Survival curves were estimated using the Kaplan-Meier method and plotted. Candidate predictor variables were tested at $P<0.05$ by univariate and multivariate Cox Regression using the $z$-score corresponding to the hazard ratio.

Data availability. Microarray data were deposited at the NCBI Gene Expression Omnibus (http://www.ncbi.nlm.nih.gov/geo) under accession number GSE37994. All data sets are available from the authors upon request.

Received: 28 July 2016 Accepted: 21 September 2017

Published online: 24 November 2017

\section{References}

1. Kopan, R. \& Ilagan, M. X. The canonical Notch signaling pathway: unfolding the activation mechanism. Cell 137, 216-233 (2009).

2. Blanpain, C., Lowry, W. E., Pasolli, H. A. \& Fuchs, E. Canonical notch signaling functions as a commitment switch in the epidermal lineage. Genes Dev. 20, 3022-3035 (2006).

3. Rangarajan, A. et al. Notch signaling is a direct determinant of keratinocyte growth arrest and entry into differentiation. EMBO J. 20, 3427-3436 (2001).

4. Ohashi, S. et al. NOTCH1 and NOTCH3 coordinate esophageal squamous differentiation through a CSL-dependent transcriptional network. Gastroenterology 139, 2113-2123 (2010).

5. Demehri, S., Turkoz, A. \& Kopan, R. Epidermal Notch1 loss promotes skin tumorigenesis by impacting the stromal microenvironment. Cancer Cell 16, 55-66 (2009).

6. Agrawal, N. et al. Exome sequencing of head and neck squamous cell carcinoma reveals inactivating mutations in NOTCH1. Science 333, 1154-1157 (2011).

7. Stransky, N. et al. The mutational landscape of head and neck squamous cell carcinoma. Science 333, 1157-1160 (2011).

8. Dotto, G. P. \& Rustgi, A. K. Squamous cell cancers: a unified perspective on biology and genetics. Cancer Cell 29, 622-637 (2016).

9. Nicolas, M. et al. Notch1 functions as a tumor suppressor in mouse skin. Nat. Genet. 33, 416-421 (2003).

10. Zhong, R. et al. Notch1 activation or loss promotes HPV-induced oral tumorigenesis. Cancer Res. 75, 3958-3969 (2015).

11. Ohashi, S. et al. A NOTCH3-mediated squamous cell differentiation program limits expansion of EMT-competent cells that express the ZEB transcription factors. Cancer Res. 71, 6836-6847 (2011).

12. Kagawa, S. et al. Cellular senescence checkpoint function determines differential Notch1-dependent oncogenic and tumor-suppressor activities. Oncogene 34, 2347-2359 (2015). 
13. Yuan, X. et al. Notch signaling: an emerging therapeutic target for cancer treatment. Cancer Lett. 369, 20-27 (2015).

14. Ronchini, C. \& Capobianco, A. J. Induction of cyclin D1 transcription and CDK2 activity by Notch(ic): implication for cell cycle disruption in transformation by Notch(ic). Mol. Cell Biol. 21, 5925-5934 (2001).

15. Zavadil, J., Cermak, L., Soto-Nieves, N. \& Bottinger, E. P. Integration of TGFbeta/Smad and Jagged1/Notch signalling in epithelial-to-mesenchymal transition. EMBO J. 23, 1155-1165 (2004).

16. Niessen, K. et al. Slug is a direct Notch target required for initiation of cardiac cushion cellularization. J. Cell Biol. 182, 315-325 (2008).

17. Sahlgren, C., Gustafsson, M. V., Jin, S., Poellinger, L. \& Lendahl, U. Notch signaling mediates hypoxia-induced tumor cell migration and invasion. Proc. Natl. Acad. Sci. USA 105, 6392-6397 (2008).

18. Ansieau, S. et al. Induction of EMT by twist proteins as a collateral effect of tumor-promoting inactivation of premature senescence. Cancer Cell 14, 79-89 (2008).

19. Ohashi, S. et al. Epidermal growth factor receptor and mutant p53 expand an esophageal cellular subpopulation capable of epithelial-to-mesenchymal transition through ZEB transcription factors. Cancer Res. 70, 4174-4184 (2010).

20. Ohashi, S. et al. Recent advances from basic and clinical studies of esophageal squamous cell carcinoma. Gastroenterology 149, 1700-1715 (2015).

21. Basu, D. et al. Evidence for mesenchymal-like sub-populations within squamous cell carcinomas possessing chemoresistance and phenotypic plasticity. Oncogene 29, 4170-4182 (2010).

22. Uchikado, Y. et al. Slug expression in the E-cadherin preserved tumors is related to prognosis in patients with esophageal squamous cell carcinoma. Clin. Cancer Res. 11, 1174-1180 (2005).

23. Usami, Y. et al. Snail-associated epithelial-mesenchymal transition promotes oesophageal squamous cell carcinoma motility and progression. J. Pathol. 215, 330-339 (2008).

24. Mani, S. A. et al. The epithelial-mesenchymal transition generates cells with properties of stem cells. Cell 133, 704-715 (2008).

25. Celia-Terrassa, T. et al. Epithelial-mesenchymal transition can suppress major attributes of human epithelial tumor-initiating cells. J. Clin. Invest. 122, 1849-1868 (2012).

26. Al-Hajj, M., Wicha, M. S., Benito-Hernandez, A., Morrison, S. J. \& Clarke, M. F. Prospective identification of tumorigenic breast cancer cells. Proc. Natl. Acad. Sci. USA 100, 3983-3988 (2003).

27. Zhao, J. S. et al. Tumor initiating cells in esophageal squamous cell carcinomas express high levels of CD44. PLoS ONE 6, e21419 (2011).

28. Biddle, A. et al. Cancer stem cells in squamous cell carcinoma switch between two distinct phenotypes that are preferentially migratory or proliferative. Cancer Res. 71, 5317-5326 (2011).

29. Prince, M. E. et al. Identification of a subpopulation of cells with cancer stem cell properties in head and neck squamous cell carcinoma. Proc. Natl. Acad. Sci. USA 104, 973-978 (2007)

30. Whelan, K. A. et al. Autophagy supports generation of cells with high CD44 expression via modulation of oxidative stress and Parkin-mediated mitochondrial clearance. Oncogene 36, 4843-4858 (2017).

31. Kinugasa, H. et al. Mitochondrial SOD2 regulates epithelial-mesenchymal transition and cell populations defined by differential CD44 expression. Oncogene 34, 5229-5239 (2015).

32. Zavadil, J. \& Bottinger, E. P. TGF-beta and epithelial-to-mesenchymal transitions. Oncogene 24, 5764-5774 (2005).

33. Brabletz, S. et al. The ZEB1/miR-200 feedback loop controls Notch signalling in cancer cells. EMBO J. 30, 770-782 (2011).

34. Postigo, A. A. Opposing functions of ZEB proteins in the regulation of the TGFbeta/BMP signaling pathway. EMBO J. 22, 2443-2452 (2003).

35. Postigo, A. A., Depp, J. L., Taylor, J. J. \& Kroll, K. L. Regulation of Smad signaling through a differential recruitment of coactivators and corepressors by ZEB proteins. EMBO J. 22, 2453-2462 (2003).

36. Vallejo, D. M., Caparros, E. \& Dominguez, M. Targeting Notch signalling by the conserved miR-8/200 microRNA family in development and cancer cells. EMBO J. 30, 756-769 (2011).

37. Zhang, T. et al. A genetic cell context-dependent role for ZEB1 in lung cancer. Nat. Commun. 7, 12231 (2016).

38. Wang, T. et al. Notch-1-mediated esophageal carcinoma EC-9706 cell invasion and metastasis by inducing epithelial-mesenchymal transition through Snail. Tumour Biol. 35, 1193-1201 (2014).

39. Tang, X. H., Scognamiglio, T. \& Gudas, L. J. Basal stem cells contribute to squamous cell carcinomas in the oral cavity. Carcinogenesis 34, 1158-1164 (2013).

40. Chang, C. J. et al. p53 regulates epithelial-mesenchymal transition and stem cell properties through modulating miRNAs. Nat. Cell Biol. 13, 317-323 (2011).

41. Lefort, K. et al. Notch1 is a p53 target gene involved in human keratinocyte tumor suppression through negative regulation of ROCK1/2 and MRCKalpha kinases. Genes Dev. 21, 562-577 (2007).
42. Maltzman, W. \& Czyzyk, L. UV irradiation stimulates levels of p53 cellular tumor antigen in nontransformed mouse cells. Mol. Cell Biol. 4, 1689-1694 (1984).

43. Yugawa, T. et al. Regulation of Notch1 gene expression by p53 in epithelial cells. Mol. Cell Biol. 27, 3732-3742 (2007).

44. Maillard, I. et al. Mastermind critically regulates Notch-mediated lymphoid cell fate decisions. Blood 104, 1696-1702 (2004).

45. Blokzijl, A. et al. Cross-talk between the Notch and TGF-beta signaling pathways mediated by interaction of the Notch intracellular domain with Smad3. J. Cell Biol. 163, 723-728 (2003).

46. Nam, J. S. et al. An anti-transforming growth factor beta antibody suppresses metastasis via cooperative effects on multiple cell compartments. Cancer Res. 68, 3835-3843 (2008).

47. Ovcharenko, I., Nobrega, M. A., Loots, G. G. \& Stubbs, L. ECR Browser: a tool for visualizing and accessing data from comparisons of multiple vertebrate genomes. Nucleic Acids Res. 32, W280-W286 (2004).

48. Cui, H., Kong, Y., Xu, M. \& Zhang, H. Notch3 functions as a tumor suppressor by controlling cellular senescence. Cancer Res. 73, 3451-3459 (2013).

49. Bao, B. et al. Notch-1 induces epithelial-mesenchymal transition consistent with cancer stem cell phenotype in pancreatic cancer cells. Cancer Lett. 307, 26-36 (2011)

50. Wellner, U. et al. The EMT-activator ZEB1 promotes tumorigenicity by repressing stemness-inhibiting microRNAs. Nat. Cell Biol. 11, 1487-1495 (2009).

51. Rhim, A. D. et al. EMT and dissemination precede pancreatic tumor formation. Cell 148, 349-361 (2012).

52. Pickup, M., Novitskiy, S. \& Moses, H. L. The roles of TGF $\beta$ in the tumour microenvironment. Nat. Rev. Cancer 13, 788-799 (2013).

53. Osipo, C., Golde, T. E., Osborne, B. A. \& Miele, L. A. Off the beaten pathway: the complex cross talk between Notch and NF-kappaB. Lab. Invest. 88, 11-17 (2008).

54. Gu, F. et al. Expression of Stat3 and Notch1 is associated with cisplatin resistance in head and neck squamous cell carcinoma. Oncol. Rep. 23, 671-676 (2010).

55. Hijioka, H. et al. Upregulation of Notch pathway molecules in oral squamous cell carcinoma. Int. J. Oncol. 36, 817-822 (2010).

56. Zagouras, P., Stifani, S., Blaumueller, C. M., Carcangiu, M. L. \& ArtavanisTsakonas, S. Alterations in Notch signaling in neoplastic lesions of the human cervix. Proc. Natl. Acad. Sci. USA 92, 6414-6418 (1995).

57. Chaffer, C. L. et al. Poised chromatin at the ZEB1 promoter enables breast cancer cell plasticity and enhances tumorigenicity. Cell 154, 61-74 (2013).

58. Zhang, J. et al. TGF-beta-induced epithelial-to-mesenchymal transition proceeds through stepwise activation of multiple feedback loops. Sci. Signal. 7 , ra91 (2014).

59. Lu, M., Jolly, M. K., Levine, H., Onuchic, J. N. \& Ben-Jacob, E. MicroRNAbased regulation of epithelial-hybrid-mesenchymal fate determination. Proc. Natl. Acad. Sci. USA 110, 18144-18149 (2013).

60. Naganuma, S. et al. Notch receptor inhibition reveals the importance of cyclin D1 and Wnt signaling in invasive esophageal squamous cell carcinoma. Am. J. Cancer Res. 2, 459-475 (2012).

61. Alcolea, M. P. et al. Differentiation imbalance in single oesophageal progenitor cells causes clonal immortalization and field change. Nat. Cell Biol. 16, 615-622 (2014).

62. Li, K. et al. Modulation of Notch signaling by antibodies specific for the extracellular negative regulatory region of NOTCH3. J. Biol. Chem. 283, 8046-8054 (2008).

63. Wu, Y. et al. Therapeutic antibody targeting of individual Notch receptors. Nature 464, 1052-1057 (2010).

64. Teicher, B. A. Transforming growth factor-beta and the immune response to malignant disease. Clin. Cancer Res. 13, 6247-6251 (2007).

65. Whelan, K. A. et al. Autophagy mediates epithelial cytoprotection in eosinophilic oesophagitis. Gut 66, 1197-1207 (2016).

66. Basu, D. et al. EGFR inhibition promotes an aggressive invasion pattern mediated by mesenchymal-like tumor cells within squamous cell carcinomas. Mol. Cancer Ther. 12, 2176-2186 (2013).

67. Lee, J. J. et al. Hypoxia activates the cyclooxygenase-2-prostaglandin E synthase axis. Carcinogenesis 31, 427-434 (2010).

68. Liu, K. et al. Sox2 cooperates with inflammation-mediated Stat3 activation in the malignant transformation of foregut basal progenitor cells. Cell Stem Cell 12, 304-315 (2013).

69. Yang, Y. \& Katz, J. P. KLF4 is downregulated but not mutated during human esophageal squamous cell carcinogenesis and has tumor stage-specific functions. Cancer Biol. Ther. 17, 422-429 (2016).

70. Griffin, M. C., Robinson, R. A. \& Trask, D. K. Validation of tissue microarrays using p53 immunohistochemical studies of squamous cell carcinoma of the larynx. Mod. Pathol. 16, 1181-1188 (2003). 
71. Stairs, D. B. et al. Deletion of p120-catenin results in a tumor microenvironment with inflammation and cancer that establishes it as a tumor suppressor gene. Cancer Cell 19, 470-483 (2011).

72. Olive, K. P. et al. Mutant p53 gain of function in two mouse models of LiFraumeni syndrome. Cell 119, 847-860 (2004).

73. Tang, X. H., Knudsen, B., Bemis, D., Tickoo, S. \& Gudas, L. J. Oral cavity and esophageal carcinogenesis modeled in carcinogen-treated mice. Clin. Cancer Res. 10, 301-313 (2004).

74. Kalabis, J. et al. Isolation and characterization of mouse and human esophageal epithelial cells in 3D organotypic culture. Nat. Protoc. 7, 235-246 (2012).

75. Facompre, N. D. et al. JARID1B enables transit between distinct states of the stem-like cell population in oral cancers. Cancer Res. 76, 5538-5549 (2016).

76. Jeffries, S. \& Capobianco, A. J. Neoplastic transformation by Notch requires nuclear localization. Mol. Cell Biol. 20, 3928-3941 (2000).

77. Sanjana, N. E., Shalem, O. \& Zhang, F. Improved vectors and genome-wide libraries for CRISPR screening. Nat. Methods 11, 783-784 (2014).

78. Takaoka, M. et al. Ha-Ras(G12V) induces senescence in primary and immortalized human esophageal keratinocytes with p53 dysfunction. Oncogene 23, 6760-6768 (2004).

79. Kim, S.-H. et al. Tumorigenic conversion of primary human esophageal epithelial cells using oncogene combinations in the absence of exogenous Ras. Cancer Res. 66, 10415-10424 (2006).

80. Natsuizaka, M. et al. Insulin-like growth factor-binding protein-3 promotes transforming growth factor-\{beta\}1-mediated epithelial-to-mesenchymal transition and motility in transformed human esophageal cells. Carcinogenesis 31, 1344-1353 (2010)

81. Nishihira, T., Hashimoto, Y., Katayama, M., Mori, S. \& Kuroki, T. Molecular and cellular features of esophageal cancer cells. J. Cancer Res. Clin. Oncol. 119, 441-449 (1993).

82. Sashiyama, H. et al. Immortalization of human esophageal keratinocytes by E6 and E7 of human papillomavirus type 16. Int. J. Oncol. 19, 97-103 (2001).

83. Harada, H. et al. Telomerase induces immortalization of human esophageal keratinocytes without p16INK4a inactivation. Mol. Cancer Res. 1, 729-738 (2003).

84. DeWard, A. D., Cramer, J. \& Lagasse, E. Cellular heterogeneity in the mouse esophagus implicates the presence of a nonquiescent epithelial stem cell population. Cell Rep. 9, 701-711 (2014).

85. Tanaka, K. et al. ALDH2 modulates autophagy flux to regulate acetaldehydemediated toxicity thresholds. Am. J. Cancer Res. 6, 781-796 (2016).

86. Natsuizaka, M. et al. IGFBP3 promotes esophageal cancer growth by suppressing oxidative stress in hypoxic tumor microenvironment. Am. J. Cancer Res. 4, 29-41 (2014).

87. Drake, J. M. et al. ZEB1 coordinately regulates laminin-332 and $\{$ beta 4 integrin expression altering the invasive phenotype of prostate cancer cells. J. Biol. Chem. 285, 33940-33948 (2010).

88. Yashiro-Ohtani, Y. et al. Pre-TCR signaling inactivates Notch1 transcription by antagonizing E2A. Genes Dev. 23, 1665-1676 (2009).

\section{Acknowledgements}

We thank Ben Rhoades, Medha Sharma, and the staff of the Molecular Pathology and Imaging Core, Molecular Biology/Gene Expression Core, Cell Culture Core and Transgenic and Chimeric Mouse Core Facilities for technical support. We thank members of the Nakagawa lab, the Rustgi lab, and the Basu lab for helpful discussions. P.M.C. is currently in the Graduate program in Cell Biology, Physiology, and Metabolism at the University of Pennsylvania. This study was supported by the following National Institute of Health (NIH) Grants: National Cancer Institute, P01CA098101 (to H.N., M.N., K.A. W., S.K., K.T., P.M.C., A.L., V.G., D.B., E.P.W., S.N., A.J.K.-S., J.A.D., A.J.B., K.-K.W., A.K.R.), F32CA174176 (K.A.W.), F30CA175133 (A.L.), T32 (CA115299-06 to A.L.);
National Institute of Dental \& Craniofacial Research (R21DE024396 to D.B. and H.N.); and the National Institute of Diabetes and Digestive and Kidney Diseases (NIDDK) R01DK114436 (H.N.), R01DK069984 (J.P.K.), R01DK101294 (J.P.K.), R01DK100342 (J.Q.), K01DK103953 (K.A.W.), T32DK007066 (K.A.W.) and P30-DK050306 Center of Molecular Studies in Digestive and Liver Diseases, The Molecular Pathology and Imaging, Molecular Biology/Gene Expression and Cell Culture Core Facilities; and K26RR032714 (H.N.) and P30-ES013508 University of Pennsylvania Center of Excellence in Environmental Toxicology (H.N.). Additional support was provided by American Cancer Society Grant (RP-10-033-01-CCE to A.K.R.), Fonds de Recherche en Santé du Québec (P-Giroux-27692) to V.G., NYSTEM (C029555) to J.Q. and Raptor Pharmaceuticals (H.N.). K.T. is a recipient of the Japan Society for the Promotion of Science Postdoctoral Fellowship.

\section{Author contributions}

M.N., K.A.W., and H.N. designed and performed the majority of the experiments, analyzed the data, prepared the figures, and wrote the paper. M.N. generated and validated DNA constructs. M.N., S.K., K.T., P.M.C., and V.S. performed cell culture, transfection assays, retrovirus/lentivirus-mediated gene transfer, ChIP assays, flow cytometry, quantitative RT-PCR, immunoblotting, and mouse xenograft experiments K.T., K.A.W., A.L., and V.G. performed 4NQO-induced carcinogenesis experiments. K. T., V.G., and K.A.W. generated and analyzed murine esophageal organoids. K.T., S.N., and K.A.W. performed IHC and IF. J.Q. and D.S.D. developed, validated, and provided $K 5 C r e^{E R T 2} ; p 53^{\text {loxP/loxP }}$ mice and anti-ZEB1 antibody, respectively. Y.Y. and J.P.K. carried out DNA sequencing of human tumor samples. D.B., Y.K., and S.N. provided human tumor samples and analyzed clinical databases. A.J.K.-S. and S.N. reviewed and scored all immunohistochemistry samples. J.A.D., A.J.B., K.-K.W., and A.K.R. supervised the project and reviewed and edited the paper. E.P.W. performed biostatistics in experimental design and data analyses.

\section{Additional information}

Supplementary Information accompanies this paper at doi:10.1038/s41467-017-01500-9.

Competing interests: The authors declare no competing financial interests.

Reprints and permission information is available online at http://npg.nature.com/ reprintsandpermissions/

Publisher's note: Springer Nature remains neutral with regard to jurisdictional claims in published maps and institutional affiliations.

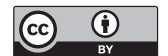

Open Access This article is licensed under a Creative Commons Attribution 4.0 International License, which permits use, sharing, adaptation, distribution and reproduction in any medium or format, as long as you give appropriate credit to the original author(s) and the source, provide a link to the Creative Commons license, and indicate if changes were made. The images or other third party material in this article are included in the article's Creative Commons license, unless indicated otherwise in a credit line to the material. If material is not included in the article's Creative Commons license and your intended use is not permitted by statutory regulation or exceeds the permitted use, you will need to obtain permission directly from the copyright holder. To view a copy of this license, visit http://creativecommons.org/ licenses/by/4.0/

(C) The Author(s) 2017 